
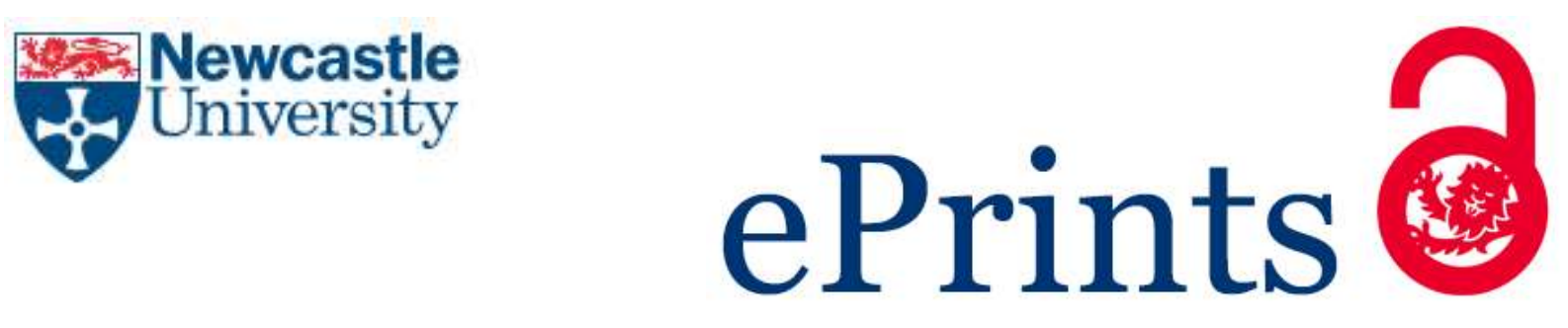

Shi W, Atlar M, Rosli R, Aktas B, Norman R. Cavitation observations and noise measurements of horizontal axis tidal turbines with biomimetic blade leading-edge designs. Ocean Engineering 2016, 121, 143-155.

\title{
Copyright:
}

(C) 2016. This manuscript version is made available under the CC-BY-NC-ND 4.0 license

DOI link to article:

http://dx.doi.org/10.1016/j.oceaneng.2016.05.030

Date deposited:

05/07/2016

Embargo release date:

26 May 2017

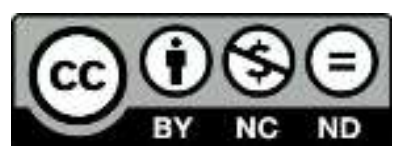

This work is licensed under a

Creative Commons Attribution-NonCommercial-NoDerivatives 4.0 International licence 


\section{Cavitation observations and noise measurements of horizontal axis tidal turbines with biomimetic blade leading- edge designs}

Weichao Shi ${ }^{* 1}$, Mehmet Atlar ${ }^{1}$, Roslynna Rosli ${ }^{1}$, Batuhan Aktas ${ }^{1}$, Rosemary Norman ${ }^{1}$

1 School of Marine Science and Technology, Newcastle University, UK

Corresponding Author:

Weichao Shi, w.shi6@newcastle.ac.uk

School of Marine Science and Technology

Armstrong Building, Newcastle University

United Kingdom, NE1 7RU

Tel: 0044 (0)191 2226726

Fax: $0044(0) 1912225491$ 
Abstract: This paper focuses on the study of cavitation and underwater noise performance of a biomimetically improved horizontal axis tidal turbine (HATT) with a leading edge design inspired by the tubercles on the pectoral fins of humpback whales. Systematic model tests were recently conducted and details of this test campaign together with the findings are summarised in the paper.

Several full-scale tidal turbine application cases were studied to understand the full-scale operating conditions considering the characteristics of varied kinds of tidal energy devices, the varying wave height and the flood/ebb tide. A systematic test regime was then designed and conducted. A set of tidal turbines with different leading-edge profiles was manufactured and tested under different loading and hence cavitation conditions. During the tests, cavitation was observed and underwater noise level was measured in comparison with the cavitation and noise performance of a counterpart HATT without tubercles.

The tested turbines displayed two main types of cavitation patterns independent of the tubercles. These were steady tip vortex cavitation and relatively intermittent cloud cavitation with a misty appearance. The leading-edge tubercles triggered the cavitation onset earlier for the tidal turbine but constrained the cavitation region to the trough between tubercles with a lesser extent on the blades. The noise performance was strongly related to the blade cavitation hence it was influenced by the leading-edge tubercles. While the turbine was working under the noncavitating conditions the total noise level was similar to the background noise level. With the increase of the tip speed ratio the noise level was increased, while increasing blade pitch angle reduced the noise level due to lower blade loading. Cavitation inception and noise diagrams are provided as a database for future studies.

Keywords: Horizontal axis tidal turbine, Leading-edge tubercle, Model tests, Cavitation observations, Underwater radiated noise measurements, 


\section{Introduction}

Tidal energy is a form of marine renewable energy used to generate electricity through turbines driven by the twice daily rising and falling of the tide. It is an attractive form of renewable energy, which is highly reliable, predictable and abundant in coastal regions (Charlier, 2003; Li et al., 2010; Pelc and Fujita, 2002). Developments over the last 20 years mean that the Horizontal Axis Tidal Turbine (HATT), which is also called "the underwater wind turbine", has reached the stage that it is very close to commercial development (Bahaj et al., 2007; Khan et al., 2009). In fact, many companies, like Atlantis Resources and Alstom already have the technology to provide a tidal energy solution with ratings from 1 to 3MW (Alstom, 2013; Atlantis, 2015). The feasibility of extracting tidal energy is no longer a question. However due to the harsh sea conditions and prevalence of marine organisms, the reliability and economy of tidal energy has been questioned in the past ten years (Douglas et al., 2008; Mitchell and Connor, 2004).

As it has been commonly acknowledged, the technology of the HATT is mainly inherited from the wind turbine industry. From the turbine blade, the generator, the control system through to the grid integration system, most of the systems mimic the technology developed for wind turbines. From the power generation point of view, the main difference between wind turbines and tidal turbines is only the density difference between air and water. However, there are other issues that the wind turbines do not experience. For example, cavitation is one of the major differences between water and air. Blade cavitation has significant detrimental effects on the hydrodynamic and structural performance of tidal turbines. First of all, it can lead to a significant loss of efficiency. Secondly, it causes erosion on the turbine blade which would cause significant damage to the blade structure as the resin in composite material, which most turbine blades are made of, can't resist the erosion resulting from cavitation (Harper and Hallett, 2015).

However, to-date few studies have looked into this area. In 2007, Bahaj et al. and Wang et al. published two papers regarding tidal turbine cavitation (Bahaj et al., 2007; Wang et al., 2007). They independently observed the cavitation patterns on two different tidal turbines in two different research facilities, which proves the existence of cavitation on tidal turbines.

Meanwhile, the magnitude of noise generated by the blade cavitation is also much higher than the turbulence noise generated by the turbine itself. There is a growing environmental awareness around various anthropogenic activities and their potential impact on marine fauna however, whilst there is a plethora of research activities taking place in terms of offshore wind turbine noise (Madsen et al., 2006), piling noise (Mueller-Blenkle et al., 2010) and shipping noise (Aktas et al., 2015; Hildebrand, 2009), the existing research regarding HATT noise is very scarce. It has been demonstrated that human activities have significant impacts on marine fauna and therefore it was considered essential to conduct a study into the underwater noise properties of a tidal energy device (Simmonds et al., 2004).

Under the above circumstances, continuing the design and optimisation work on tidal turbine blades started by Wang et al. (2007), the team in the Emerson Cavitation Tunnel (ECT), Newcastle University has recently initiated a new turbine blade study, by applying the leadingedge tubercles of humpback whale flippers to a tidal turbine (Shi et al., 2016a; Shi et al., 2016b). The tubercles have the potential to improve the performance of foils. Their benefits were first investigated in wind tunnel tests on a pair of replica humpback whale flippers with and without leading-edge tubercles (Miklosovic et al., 2007; Miklosovic et al., 2004). The experiments 
demonstrated a delayed stall and also enhanced lift-to-drag ratio. After that, many research studies, both numerical and experimental in nature, have investigated the potential applications of leading-edge tubercles as applied on air fans, wind turbines, rudders and so on (Corsini et al., 2013; Howle, Jan 24,2009; Swanson and Isaac, 2011; van Nierop et al., 2008; Weber et al., 2010). A study was also conducted recently to improve a tidal turbine's performance by applying tubercles to the blades and performance comparisons of tidal turbine models with different tubercle designs were carried out in a towing tank (Gruber et al., 2011). This work demonstrated the great potential to apply tubercles to tidal turbines. The power coefficient of the turbines with tubercles showed a significant enhancement compared to the turbine without tubercles.

In the study of applying leading-edge tubercles to a marine rudder, Weber et al. noticed that the tubercles can trigger the inception of cavitation earlier than for a conventional rudder but that they constrain the cavitation in the trough between two tubercles (Weber et al., 2010). This may also be the case for the tidal turbine with a potential reduction in cavitation extent. Bearing in mind the fact that there has been no investigation reported on the effect of tubercles on the underwater radiated noise levels of tidal turbines it was thought to be most interesting to conduct a cavitation and noise test campaign with tidal turbines to explore these aspects.

An optimisation design study of the leading-edge tubercles for a turbine blade, preliminary hydrofoil tests and hydrodynamic efficiency tests for model turbines have been conducted (Shi et al., 2015; Shi et al., 2014). It was shown that the leading-edge tubercles can help the turbine to produce more torque at lower values of Tip Speed Ratio (TSR) before the turbine reached at its maximum power coefficient $(\mathrm{Cp})$. This enhancement can help the turbine to start at a lower tidal flow velocity reducing the magnitude of the cutting-in speed without compromising the maximum $\mathrm{Cp}$.

Based on the above background, given the promising potential of applying tubercles to the turbine, an investigation was undertaken into the cavitation and noise characteristics of such a device in comparison with a counterpart HATT device without the tubercle arrangement. Within this framework, this paper firstly investigates the possible operating conditions of various HATT devices. Three tidal turbine models with different leading-edge profiles were then tested in the ECT under the simulated operating conditions. In the remaining sections of the paper, the details of cavitation observations and noise measurements are presented and discussed.

\section{Analysis of full-scale operating conditions}

Over the last two decades, tidal energy researchers have developed various HATT devices to extract energy from the tidal current. These devices have different control systems to control either the blade pitch angle or the rotational speed to suit the changing incoming velocity in order to maintain the maximum power generating efficiency. They also have different designs of their supporting structures, such as floating platforms, fixed foundations and so on.

Because of these differences, the operating conditions of the HATTs are quite different. The blade radius, the blade pitch angle, the shaft emersion depth and also the rotating speed will all contribute in operating conditions. The non-dimensional cavitation number, which assures the hydrodynamic similarity for cavitation between two geometrically similar turbines, is therefore dependent upon these parameters. In this paper the cavitation number is defined at the top 0.7 radius of the turbine blade, $\operatorname{Cav}_{0.7 r}$ which can be described as in Equation 1. 


$$
\operatorname{Cav}_{0.7 r}=\frac{P_{0.7 r}-P_{v}}{\frac{1}{2} \rho \sqrt{\left(V^{2}+(0.7 \omega r)^{2}\right.}}
$$

Where $P_{0.7 r}$ is the static pressure at the upper 0.7 radius of the turbine including the Atmospheric pressure, $\mathrm{Pa} ; P_{v}$ is the vapour pressure of the water, $\mathrm{Pa} ; \omega$ is the rotational speed, $\mathrm{rad} / \mathrm{s} ; \rho$ is the water density, $\mathrm{kg} / \mathrm{m}^{3} ; V$ is the incoming velocity, $\mathrm{m} / \mathrm{s} ; r$ is the turbine radius, $\mathrm{m}$.

The $\operatorname{Cav}_{0.7 r}$ of different designs of tidal turbines was studied and calculated to determine the test conditions for the model tests. Four typical HATT cases equipped with different control schemes and different supporting structures were studied as described in the next section.

\subsection{Designs of HATT}

\subsubsection{HATT with variable speed control and fixed foundation}

A tidal turbine with variable speed control and fixed foundation is widely used in the current development of tidal current energy devices. One example is the AR1000 built by Atlantis Resources and tested at the European Marine Energy Centre (EMEC), as shown in Figure 1. The turbine has three fixed pitch blades with a diameter of $18 \mathrm{~m}$ and controlled by a variable speed control system. The AR1000 is designed to produce its nameplate capacity of $1 \mathrm{MW}$ in water flows of $2.65 \mathrm{~m} / \mathrm{s}$ and above (Atlantis, 2015). The turbine is supported by a gravity-based foundation working in around $40 \mathrm{~m}$ deep oceans. This turbine is now scheduled to be installed on CECEP's Daishan demonstration site in China.

The rotational speed of this kind of device is always being adjusted with the varying incoming flow velocity to keep the turbine working at the optimum TSR. The shaft immersion is also changing because of the tide and the waves.

\subsubsection{HATT with pitch control and fixed foundation}

The HATT with pitch control is another type of tidal turbine that is also very popular and which is very similar to the wind turbine and typically supported by a fixed structure. The pitch angle can be controlled to adapt to the varying incoming velocity. A typical device would be one produced by Alstom and also tested in the EMEC site as shown in Figure 2 (Alstom, 2013). Its rotor has three pitch controlled blades with a diameter of 18 meters. The unit operates fully submerged, with no surface piercing parts, in a water depth of about 40 meters.

Instead of maintaining the optimum TSR by changing the rotational speed, this device maintains a constant rotational speed by controlling its blade pitch angle to suit the changing incoming velocity. Because of the fixed foundation, the shaft immersion also changes with the tide and the waves.

\subsubsection{HATT with variable speed control and floating platform}

Aiming to minimise the whole lifecycle cost of electricity production from tidal energy and other reasons for justifying the deep water locations, floating platforms are becoming more and more popular in tidal energy. The SR2000 device, as shown in Figure 3, produced by Scotrenewables Tidal Power Ltd, can be installed in any water depths of $25 \mathrm{~m}$ and above due 
to the flexible mooring system. The SR2000 carries two $16 \mathrm{~m}$ fixed pitch rotors, giving a rating of $2 \times 1 \mathrm{MW}$ for a $3 \mathrm{~m} / \mathrm{s}$ current speed and using variable speed control (Scotrenewables, 2015).

Like the other devices with variable speed control, the rotational speed of this kind of device is constantly adjusting with the varying incoming velocity. However, because of its floating platform the shaft immersion does not change with the tide and the waves greatly.

\subsubsection{HATT with pitch control and floating platform}

Similarly, the floating platform can also be used to carry a HATT with pitch control. Bluewater produce a floating platform, BlueTEC, which can carry different kinds of turbine, as shown in Figure 4 . These turbines can also be controlled by pitch control systems.

The pitch control tidal turbines will maintain a constant rotational speed but change the blade pitch angle to suit the changing incoming velocity. However, the shaft immersion does not change with the tide and the waves.

\subsection{Brief introduction to selected tidal turbine}

According to the review in Section 2.1, a 1MW tidal turbine typically will have around a $20 \mathrm{~m}$ blade diameter subject to an assumed design tide (incoming flow) velocity of $3 \mathrm{~m} / \mathrm{s}$. Depending on the supporting structure, the shaft immersion may change with the tide height and the wave height. In this paper a typical three bladed HATT was chosen for the study. This turbine model was designed and tested during a previous project (Wang et al., 2007) and validated by a CFD study (Shi et al., 2013). The main parameters of the notional full-scale design are given in Table 1 .

\subsection{Operating matrix of selected tidal turbine}

Considering different working conditions, operating conditions matrices for the pitch control turbine and for the variable speed control turbine combined with different shaft immersion depths and different incoming flow speeds were generated and are given in Table 2 and Table 3 , respectively. By taking ebb tide and wave height into consideration, the shaft immersion varies from $20 \mathrm{~m}$ to $11 \mathrm{~m}$ (only $1 \mathrm{~m}$ blade tip clearance from the free surface). The incoming velocity (Vin) varies from $2 \mathrm{~m} / \mathrm{s}$ to $4.5 \mathrm{~m} / \mathrm{s}$ assuming a spring tide.

Based on the above assumptions, full-scale tidal turbine operating conditions were used to arrange the model tests. Because of the large number of test conditions, a systematic test regime was arranged to plot a cavitation diagram in order to cover a reasonable range of cavitation numbers. 


\section{Experimental set-up and conditions}

\subsection{Tidal turbine models with leading-edge tubercles}

Based on the previous numerical optimisation and experimental validation study (Shi et al., 2015, 2016a), the sinusoidal leading-edge profile was defined as shown in Figure 5. The amplitude (A) of the sinusoidal tubercles was equal to $10 \%$ of the local chord length (C) while eight tubercles were evenly distributed along the radius with the wavelength $(\mathrm{W})$ equal to $20 \mathrm{~mm}$. The profile of the leading edge tubercles was as represented by Equation 2.

$$
H=\frac{A}{2} \operatorname{Cos}\left[\frac{2 \pi}{W}(r-40)-\pi\right]+\frac{A}{2}
$$

Equation 2

Where $\mathrm{H}$ is the height of the leading-edge profile relative to the reference one which is the smooth leading-edge profile.

Three pitch-adjustable turbine models with different leading-edge profiles were manufactured by Centrum Techniki Okrętowej S.A. (CTO, Gdansk), as shown in Figure 6. The radial chord and pitch distribution for the blades of this $400 \mathrm{~mm}$ diameter model turbine are shown in Table 4. The S814 airfoil profile was chosen as the main blade section as shown in Figure 7. The turbine model without tubercles is referred to as the reference (or basis) turbine and denoted by "Ref" while the one with two leading-edge tubercles at the tip is denoted by "Sin_2" and with eight leading-edge tubercles by "Sin_8", as shown in Figure 6.

\subsection{Experimental setup}

\subsubsection{Brief introduction to testing facility}

The models were tested in the Emerson Cavitation Tunnel (ECT) at Newcastle University. The outline of the tunnel is shown in Figure 8. The tunnel is a medium size propeller cavitation tunnel with a measuring section of $1219 \mathrm{~mm} \times 806 \mathrm{~mm}$ (width $\times$ height) (Atlar, 2011).

The turbine was mounted on a dynamometer, $K \& \mathrm{R} \mathrm{H33}$, designed to measure the thrust and torque of a propeller or turbine. A DC motor $(64 \mathrm{~kW}$ at $415 \mathrm{~V})$ is mounted on top of the dynamometer to drive the turbine and control its rotational speed for the purpose of achieving the desired TSR, as defined in Equation 3.

$$
T S R=\frac{\omega r}{V}
$$

Equation 3

where $\omega$ is the rotational speed, $\mathrm{rad} / \mathrm{s} ; \mathrm{V}$ is the tunnel flow velocity, $\mathrm{m} / \mathrm{s} ; \mathrm{r}$ is the turbine radius, $\mathrm{m}$.

\subsubsection{Image processing}

Two high-speed cameras (NanoSense MK III) and a still DSLR camera (Nikon D90) were used to capture the moving and still images of the cavitation patterns, respectively. During the tests 
the strobe lights and high-speed cameras were triggered by the encoder, in phase-lock mode, in order to freeze the image recordings to be always in the same blade position.

\subsubsection{Noise measurement}

The noise measurements were captured by using a Bruel \& Kjaer $(\mathrm{B} \& \mathrm{~K})$ data acquisition system and B\&K Type 8103 hydrophone which was installed in a specially designed fin located in the tunnel at a distance of $438 \mathrm{~mm}$ from the model turbine, as shown in Figure 9.

The noise signals were then processed using the PULSE lab-shop software CPB and FFT analysers. The captured signals, which are in $1 \mathrm{~Hz}$ and $1 / 3$ octave bands, were averaged over 50 seconds to eliminate the effect of any instantaneous noise sources. The settings in Table 5 were used with the 8103 hydrophone.

Following the recommendations of the $15^{\text {th }}$ ITTC Cavitation Committee, the measured Sound Pressure Levels (SPL) were corrected to an equivalent $1 \mathrm{~Hz}$ bandwidth and $1 \mathrm{~m}$ source level using Equation 4 (ITTC, 1978).

$$
S P L=S P L_{m}-10 \log \Delta f+20 \log (r)
$$

Equation 4

Where $S P L$ is the Sound Pressure level in $1 \mathrm{~Hz}$ band in dB relative to $1 \mu \mathrm{Pa}$ at $1 \mathrm{~m} ; S P L_{m}$ is the Sound Pressure level in $1 / 3$ Octave band in $\mathrm{dB}$ relative to $1 \mu \mathrm{Pa} ; \Delta f$ is the frequency bandwidth for the 1/3 Octave band for each centre frequency; $r$ is the distance of the location of the hydrophone from the turbine centreline.

In order to achieve the net noise sound pressure level $\left(S P L_{N}\right)$ at each centre frequency, Equation 5 is used to subtract the background noise $\left(S P L_{B}\right)$ from the total noise level $\left(S P L_{T}\right)$.

$$
S P L_{N}=10 \log \left[10^{\left(S P L_{T} / 10\right)}-10^{\left(S P L_{B} / 10\right)}\right]
$$

Equation 5

\subsection{Test matrix}

A systematic test matrix was arranged for the model experiments to consider the various parameters discussed earlier, as shown in Table 6. Three different incoming flow velocities were used and the rotational speed was varied accordingly to achieve the desired TSRs. By applying the Atmospheric pressure on the tunnel the range of the cavitation numbers, which was applied in the test programme, covered the full-scale operating conditions, as shown in Figure 11. Three different pitch angles were also applied to understand the effect of pitch on the cavitation performance. 


\section{Results}

\subsection{Cavitation observations}

In this section, the results of the cavitation observation are presented. First the comparison is made between the three different turbine models in order to find out the difference in the cavitation patterns caused by the leading-edge tubercles. Then, based on the experimental observations, a cavitation diagram is presented to provide a prediction for the full-scale operations.

\subsubsection{Typical cavitation patterns}

During the tests, two main types of cavitation pattern were noted once the cavitation was incepted. These were tip vortex cavitation and cloud cavitation with a misty appearance at the back or face side of the blade depending on the TSRs. The development sequence of these cavitation types on the blades was such that first the tip vortex cavitation appeared due to the higher resultant velocity at the tip in a steady manner. Then the tip vortex cavitation was gradually accompanied by a rather misty appearance of unsteady cloud cavitation on either side of the turbine blade depending on the TSR. While the cloud cavitation would affect the turbine efficiency, the tip vortex cavitation did not have much impact on it.

\subsubsection{Tip vortex cavitation}

As presented in Table 7 (Pitch angle $=+4^{\circ} ; \mathrm{V}=3 \mathrm{~m} / \mathrm{s} ; \mathrm{TSR}=4 ; \mathrm{Cav}_{0.75 \mathrm{r}}=3.5$ ) the tip vortex cavitation was the first and most common type cavitation observed during the tests. This type of cavitation is also quite commonly observed in full-scale operation. Either a higher incoming velocity or ebb tide can trigger the tip vortex cavitation as well as extreme wave actions. During the tests it was noted that this cavitation was incepted downstream of the blades and then rapidly attached to all blade tips with increased loading in a rather steady and persistent manner. Once the vortices were attached and established at the tips it was noted that the nature (cavity dynamics) and size (diameter) of the vortices for the three different leading-edge profiles tested were quite similar in appearance.

\subsubsection{Back side cloud cavitation}

As shown in Table 8, the tip vortex cavitation could be accompanied with a misty type cloud cavitation as the loading condition deteriorates (e.g. Pitch angle $=+4^{\circ} ; \mathrm{V}=4 \mathrm{~m} / \mathrm{s} ; \mathrm{TSR}=4$; $\mathrm{Cav}_{0.75 \mathrm{r}}=1.5$ ). This is the most severe condition that has been tested for the design TSR which corresponds to an extreme condition that a variable speed controlled turbine is working under $11 \mathrm{~m}$ shaft immersion depth only $1 \mathrm{~m}$ tip clearance and $4.5 \mathrm{~m} / \mathrm{s}$ incoming velocity. This kind of cavitation is always observed at lower TSRs and lower pitch angle settings.

The observed cavitation was a cloud type cavitation but its nature was somehow different to classical cloud cavitation which has clear and relatively large bubbles. Instead it had a misty appearance composed of many micro-bubbles. The cavitation patterns of this type of cavitation were quite different for the different leading-edge designs. For the reference turbine, without leading-edge tubercles, the cavitation started from the leading edge and spread between $0.8 \mathrm{r}$ and 0.9r. Likewise for the turbines with the tubercles, the cavitation also started from the leading edge. However, the tubercles helped the turbine to constrain the cavitation only to the trough areas. However, because of the higher speed and lower pressure within the trough areas, 
Sin_8 produced more cavitation in the lower radius region $(0.7 \mathrm{R})$ compared to the other two turbines, i.e. the Ref and Sin_2.

\subsubsection{Face side cloud cavitation}

The face side cloud cavitation, as presented in Table 9, was observed under the condition: Pitch angle $=+8^{\circ} ; \mathrm{V}=3 \mathrm{~m} / \mathrm{s} ; \mathrm{TSR}=6 ; \mathrm{Cav}_{0.75 \mathrm{r}}=1.3$. This kind of cavitation generally occured at a higher TSR and a higher pitch angle setting. As shown in Figure 11, although this condition appeared to be more severe, it is not likely to be allowed to occur for a controlled turbine in full-scale. Nevertheless, if the turbine lost control and was freely spinning, this kind of cavitation might be experienced.

Regarding the cavitation pattern, this kind of cavitation started from the maximum thickness position along the blade section chord. The difference between the cavitation patterns developed over the reference turbine and the turbines with tubercles was quite obvious and similar to the effect observed with the back side cloud cavitation. The tubercles helped the blades to constrain the cavitation development to the trough areas as it can be clearly seen in Figure 12.

\subsubsection{Double-side cloud cavitation}

Imposing more severe conditions indicated that the turbines could develop cloud cavitation on both sides (back and face) of their blades in combination. This is shown in Table 10 for an operating condition of Pitch angle $=+4^{\circ} ; \mathrm{V}=4 \mathrm{~m} / \mathrm{s} ; \mathrm{TSR}=5.5 ; \mathrm{Cav}_{0.75 \mathrm{r}}=0.86$. As for the face side cloud cavitation, this condition is not in the range of the full-scale operating conditions analysed in Section 2.3.

The influence caused by the leading-edge tubercles on this cavitation development was also very similar to that observed previously for the face side and back side cloud cavitation. Cavitation started from the maximum thickness position along the blade section chord. The tubercles helped the blades to constrain the cavitation to only to occur in the area after the trough of the leading edge and also to separate the cavitation into different regions.

\subsubsection{Cavitation inception diagram}

Following the observation and study of the cavitation patterns for the turbines, a cavitation inception diagram was devised for the different leading-edge designs and different pitch angles in order to provide a practical guideline for the full-scale operation. The diagram is shown in Figure 13 where the different types of cavitation observed are labelled. In this diagram, the tip vortex cavitation inception was assumed when the vortex was attached to the blade tip.

As shown in Figure 12, the two main types of cavitation, which are the tip vortex and misty type cloud cavitation, can be observed with the turbines tested. The latter type can be erosive as well as potentially affecting the turbine efficiency while both types may contribute into the underwater noise level.

On the other hand, as remarked earlier, even though the leading-edge tubercles can limit the cavitation area to the trough parts of the profiles, they can also trigger the cavitation inception earlier than it would occur for the turbine without leading-edge tubercles depending on the pitch angle. This is because of the higher velocity and hence lower pressure in the trough area created by the tubercles. 


\subsection{Noise measurements}

Alongside the cavitation observation tests, the underwater radiated noise (URN) levels of the subject turbines were also measured. The noise data generated within this experimental campaign was extremely large and hence to present all of the information was a challenge. Therefore, a comparison was first made between the different leading-edge designs to find out the effect of the leading-edge tubercles. These comparisons were made by using the total noise data which included the tunnel background noise due to the relative nature of analysis. However, the measured sound pressure level of the reference model turbine was further analysed to subtract the tunnel background noise and then the net noise levels were plotted to provide a bench mark database for a conventional HATT turbine model which is hardly available in open literature. All of the test cases were coded as "Model Name_Pitch Angle_Test Velocity", for example "Ref_0_2" indicated the test results for "the reference turbine model" with " 0 " pitch angle setting" tested at " $2 \mathrm{~m} / \mathrm{s}$ incoming velocity", respectively.

\subsubsection{Effect of different leading-edge profiles on URN levels}

A sampled dataset (Pitch angle $=8^{0}$ ) of this test campaign is shown in Figure 14, Figure 15 and Figure 16. Each figure presents the total URN noise level including the tunnel background noise, as raw data, that has been collected by hydrophone 8103 in $1 \mathrm{~Hz}$ band with the incoming flow set to be 2, 3 and $4 \mathrm{~m} / \mathrm{s}$ respectively. Comparisons amongst the three different blade leading-edge profiles were made under the same operating conditions with respect to noncavitating and cavitating conditions described in the following.

\subsubsection{Non-cavitating conditions}

As it can be seen in Figure 14, under the conditions starting from the lowest rotating speed of the motor (start) up to the highest condition (TSR=7), the noise levels of the three turbines with the different leading-edge profiles overlap each other. Similarly from the top three plots of Figure 15, (for TSR=1 and TSR=2) and the first plot in Figure 16 (the starting condition), the noise levels of three different turbines overlap each other and are at a relatively low level under these cavitation free conditions.

\subsubsection{Tip cavitation conditions}

However, once the cavitation was incepted, the difference between the different leading-edge profiles was revealed. It can be seen in Figure 15 (for $\mathrm{TSR}=3$ and $\mathrm{TSR}=4$ at $3 \mathrm{~m} / \mathrm{s}$ ) that the noise level of Sin2 and Sin8 in the frequency range from $1 \mathrm{KHz}$ to $2 \mathrm{KHz}$ is much higher than that for the reference turbine Ref. This was because under these two conditions the turbines with the leading-edge tubercles can trigger the tip vortex cavitation earlier than for the reference turbine without the tubercles, as also shown in the cavitation diagram in Figure 13. This difference in noise results can also been seen in Figure 16 (for TSR $=3$ and TSR=4 at 4m/s) because of the additional cloud cavitation generated by Sin2 and Sin8 while only tip vortex cavitation was generated by the Reference turbine.

\subsubsection{Cloud cavitation conditions}

For the last condition while all the three turbines were suffering from the cloud cavitation, as shown in Figure 15 (for TSR=6), the noise level of the Reference turbine in the higher frequency range from $3 \mathrm{KHz}$ onwards was higher than the turbines with the leading-edge tubercles. This was because the face side cloud cavitation that was produced by the reference turbine had a larger extent and volume than that of the cavitation produced by the turbines Sin2 
and Sin8, as shown in Table 9. In Figure 12 it can be easily seen that the face side cloud cavitation generated by the reference turbine has the largest extent while with the increase of the number of tubercles, the extent of cavitation is gradually reduced.

This phenomenon can also be seen under the condition TSR $=8$ and $\mathrm{V}=2 \mathrm{~m} / \mathrm{s}$ as illustrated in Figure 14. It can be noticed that the noise level between the Reference turbine and the turbines with tubercles has a significant difference that ranges from 10-20dB. Comparing the detailed cavitation patterns shown Figure 17, while only a very small area of cavitation can be observed in the trough regions of the tubercles, the cloud cavitation generated by the reference turbine covers a much larger extent from around 0.8Rto 0.95R. Therefore, the tubercles can significantly change the noise signature via influencing the cavitation development pattern.

Based on the analysis of the noise measurements and the correlation with the cavitation observations, it is obvious that the underwater radiated noise level is highly dependent on the cavitation and its pattern. The leading-edge tubercles can influence the noise levels of turbines through the particular cavitation pattern that they generate. However, if the turbine is working under a cavitation free condition, the difference between the turbines with and without tubercles is negligible.

\subsubsection{Benchmark noise data for a typical HATT}

Based on the investigation in Section 4.2.1, it is confirmed that the acoustic performance difference caused by the different leading-edge profiles is dominated by the cavitation patterns generated. In order to complement the earlier devised cavitation inception diagram, a noise map for the net noise levels of the reference turbine was also devised to provide benchmark data for a conventional HATT turbine model. This data together with the details of the turbine geometry (i.e. Figure 6, Table 1 and Table 4) presents an invaluable contribution to state-ofthe-art tidal turbine hydrodynamic design studies as there is hardly any data of this kind published other than limited data from Wang et al (2007).

As shown in Figure 18, Figure 19 and Figure 20, while the turbine is operating in a cavitation free condition, the total noise level is of a similar level to the background noise. Therefore, at certain frequencies the noise data is apparently missing as the net noise level is less than $3 \mathrm{~dB}$. With the development of the blade cavitation the amplitude of the net noise SPL gradually increases. The cavitation contributes more in the higher frequency range as it can be observed in Figure 20 where from $500 \mathrm{~Hz}$ onward the increase in the net noise SPLs is more obvious and gradually rises with the increased TSR and reduced cavitation number.

A cross plot given in Figure 20 is provided to demonstrate the effect of varying cavitation number on the Reference turbine SPLs by keeping the pitch angle and TSR (by shaft speed) constant (at $0^{\circ}$ and TSR4, respectively) while changing the tunnel inflow speed for 2, 3 and 4 $\mathrm{m} / \mathrm{s}$. Since the turbine was free of cavitation at $2 \mathrm{~m} / \mathrm{s}$ inflow speed (see Figure 12 ) there is no measurable SPL appearing in Figure 20. However, with increasing inflow speed (at 3 and 4 $\mathrm{m} / \mathrm{s}$ ) the turbine first developed the tip vortex cavitation at $3 \mathrm{~m} / \mathrm{s}$ which was further combined with the cloud cavitation at 4m/s. As shown in Figure 20, this reflects as 15-25dB increase in the measured SPLs for the broad range of frequencies after $300 \mathrm{~Hz}$.

Another cross plot of the measured SPLs with the Reference turbine is given in Figure 21 to demonstrate the effect of TSR (and hence cavitation number) for constant pitch and inflow speed (at $0^{\circ}$ and $4 \mathrm{~m} / \mathrm{s}$, respectively). As shown in this figure the trend is very clear as increasing the TSR (or reduced cavitation number) via increasing shaft speed resulted in increased SPLs. 
The final cross plot for the Reference turbine is shown in Figure 22 to demonstrate the effect of blade pitch angle for constant TSR $=4$. As it is clearly shown, increasing blade pitch angle resulted in decreased blade loading and hence reduced SPLs. 


\section{Conclusions}

This paper reports on recently conducted model turbine tests in a medium size cavitation tunnel to investigate the effect of leading-edge tubercles on the cavitation and underwater radiated noise performance of horizontal axis tidal turbines (HATT). The paper also provides invaluable benchmark data for the cavitation and net noise levels of a typical HATT. Based on the experimental investigations the following can be concluded:

1. Over the operating range tested the turbine models with three different leading edge profiles displayed mainly two types of cavitation patterns depending on the TSR, blade pitch and depth of the shaft submergence imposed. The observed cavitation types were restricted to the continuous tip vortex cavitation and gradually developing misty type cloud cavitation in combination with increasing blade loading. The latter type can develop on the back or face side of the blade as well as on both sides depending on the loading condition.

2. The leading-edge tubercles may trigger earlier inception for the tip vortex cavitation compared to that for the reference turbine with smooth leading edge. The strength of the tip vortex cavitation appeared to be similar for the different leading-edge profiles.

3. However, the development of the misty type cloud cavitation over the leading edge tubercles was restricted to the trough areas of the tubercles. This resulted in reduced cavitation extent and rather intermittent cavitation as opposed to larger extent and continuous appearance of the cloud cavitation observed with the reference turbine.

4. The three turbines displayed almost similar total noise levels until the cavitation was incepted. Once the cavitation was incepted, the noise levels of the turbines with tubercles are generally higher than those of the reference turbine because of the early inception of the tip cavitation. When cloud cavitation was generated, the noise levels of the turbines with the tubercles were lower than those of the Reference turbine due to the constrained development and the lesser extent of cloud cavitation.

5. Net noise levels of a typical HATT turbine model indicated that the noise level was in the comparable to the background noise level while the turbine was not cavitating. Once the cavitation was incepted, the net noise level rose dramatically in the higher frequency (broad band) range due to initially developing tip vortex and gradually combining contribution from the cloud cavitation.

6. TSR and blade pitch angle are two important parameters affecting the noise levels of a typical HATT. While the increasing TSR increased the net SPL, the increased blade pitch angle reduced the net SPLs due to the reduced blade loading. 


\section{Acknowledgments}

This research is funded by the School of Marine Science and Technology, Newcastle University and the China Scholarship Council. The financial support obtained from both establishments is gratefully acknowledged. The model turbines were kindly manufactured by CTO of Gdansk with a generous student discount. The Authors would also like to thank all the team members in the Emerson Cavitation Tunnel for their help in testing and sharing their knowledge. 


\section{Reference}

Aktas, B., Atlar, M., Turkmen, S., Shi, W., Sampson, R., Korkut, E., Fitzsimmons, P., 2015. Propeller Cavitation Noise Investigations of a Research Vessel Using Medium Size Cavitation Tunnel Tests and Full-Scale Trials. Ocean Engineering.

Alstom, 2013. Alstom produced electricity with its $1 \mathrm{MW}$ tidal turbine as a part of ETI ReDAPT project.

Atlantis, 2015. AR1000.

Atlar, M., 2011. Recent upgrading of marine testing facilities at Newcastle University, AMT'11, the second international conference on advanced model measurement technology for the EU maritime industry, pp. 4-6.

Bahaj, A.S., Batten, W.M.J., McCann, G., 2007. Experimental verifications of numerical predictions for the hydrodynamic performance of horizontal axis marine current turbines. Renewable Energy 32 (15), 2479-2490.

BlueTEC, 2015. BlueTEC Modular.

Charlier, R.H., 2003. A "sleeper" awakes: tidal current power. Renewable and Sustainable Energy Reviews 7 (6), 515-529.

Corsini, A., Delibra, G., Sheard, A.G., 2013. On the role of leading-edge bumps in the control of stall onset in axial fan blades. Journal of Fluids Engineering-Transactions of the Asme 135 (8), 081104-081104.

Douglas, C.A., Harrison, G.P., Chick, J.P., 2008. Life cycle assessment of the Seagen marine current turbine. Proceedings of the Institution of Mechanical Engineers Part M-Journal of Engineering for the Maritime Environment 222 (M1), 1-12.

Gruber, T., Murray, M.M., Fredriksson, D.W., 2011. Effect of humpback whale inspired tubercles on marine tidal turbine blades, ASME 2011 International Mechanical Engineering Congress and Exposition. American Society of Mechanical Engineers, pp. 851-857.

Harper, P.W., Hallett, S.R., 2015. Advanced numerical modelling techniques for the structural design of composite tidal turbine blades. Ocean Engineering 96, 272-283.

Hildebrand, J.A., 2009. Anthropogenic and natural sources of ambient noise in the ocean. Marine Ecology Progress Series 395 (5).

Howle, L.E., Jan 24,2009. Whalepower wenvor blade. Bellequant Engineering, PLLC.

ITTC, 1978. Proceedings of the 15th International Towing Tank Conference (ITTC). ITTC, Netherland.

Khan, M., Bhuyan, G., Iqbal, M., Quaicoe, J., 2009. Hydrokinetic energy conversion systems and assessment of horizontal and vertical axis turbines for river and tidal applications: $A$ technology status review. Applied Energy 86 (10), 1823-1835. 
Li, D., Wang, S., Yuan, P., 2010. An overview of development of tidal current in China: Energy resource, conversion technology and opportunities. Renewable and Sustainable Energy Reviews 14 (9), 2896-2905.

Madsen, P.T., Wahlberg, M., Tougaard, J., Lucke, K., Tyack, P.L., 2006. Wind turbine underwater noise and marine mammals: implications of current knowledge and data needs.

Miklosovic, D.S., Murray, M.M., Howle, L.E., 2007. Experimental evaluation of sinusoidal leading edges. Journal of Aircraft 44 (4), 1404-1408.

Miklosovic, D.S., Murray, M.M., Howle, L.E., Fish, F.E., 2004. Leading-edge tubercles delay stall on humpback whale (Megaptera novaeangliae) flippers. Physics of Fluids 16 (5), L39-L42.

Mitchell, C., Connor, P., 2004. Renewable energy policy in the UK 1990-2003. Energy Policy 32 (17), 1935-1947.

Mueller-Blenkle, C., McGregor, P.K., Gill, A.B., Andersson, M.H., Metcalfe, J., Bendall, V., Sigray, P., Wood, D.T., Thomsen, F., 2010. Effects of pile-driving noise on the behaviour of marine fish, COWRIE Ref: Fish 06-08, Technical Report

Pelc, R., Fujita, R.M., 2002. Renewable energy from the ocean. Marine Policy 26 (6), 471-479.

Scotrenewables, 2015. SR2000.

Shi, W., Atlar, M., Norman, R., Aktas, B., Turkmen, S., 2015. Biomimetic improvement for a tidal turbine blade, EWTEC 2015, Nantes, France.

Shi, W., Atlar, M., Norman, R., Aktas, B., Turkmen, S., 2016a. Numerical optimization and experimental validation for a tidal turbine blade with leading-edge tubercles. Renewable Energy.

Shi, W., Atlar, M., Norman, R., Seo, K.-C., 2014. CFD investigations on leading-edge tubercles as applied on a tidal turbine blade, GRAND RENEWABLE ENERGY 2014, Tokyo, Japan.

Shi, W., Rosli, R., Atlar, M., Norman, R., Wang, D., Yang, W., 2016b. Hydrodynamic performance evaluation of a tidal turbine with leading-edge tubercles. Ocean Engineering 117, 246-253.

Shi, W., Wang, D., Atlar, M., Seo, K.-C., 2013. Flow separation impacts on the hydrodynamic performance analysis of a marine current turbine using CFD. Proceedings of the Institution of Mechanical Engineers, Part A: Journal of Power and Energy.

Simmonds, M., Dolman, S., Weilgart, L., 2004. Oceans of Noise 2004, A WDCS Science Report, WDCS, the Whale and Dolphin Conservation Society.

Swanson, T., Isaac, K.M., 2011. Biologically Inspired Wing Leading Edge for Enhanced Wind Turbine and Aircraft Performance. AIAA.

van Nierop, E., Alben, S., Brenner, M., 2008. How bumps on whale flippers delay stall: An aerodynamic model. Physical Review Letters 100 (5). 
Wang, D., Atlar, M., Sampson, R., 2007. An experimental investigation on cavitation, noise, and slipstream characteristics of ocean stream turbines. Proceedings of the Institution of Mechanical Engineers, Part A: Journal of Power and Energy 221 (2), 219-231.

Weber, P.W., Howle, L.E., Murray, M.M., 2010. Lift, drag, and cavitation onset on rudders with leading-edge tubercles. Marine Technology and Sname News 47 (1), 27-36. 


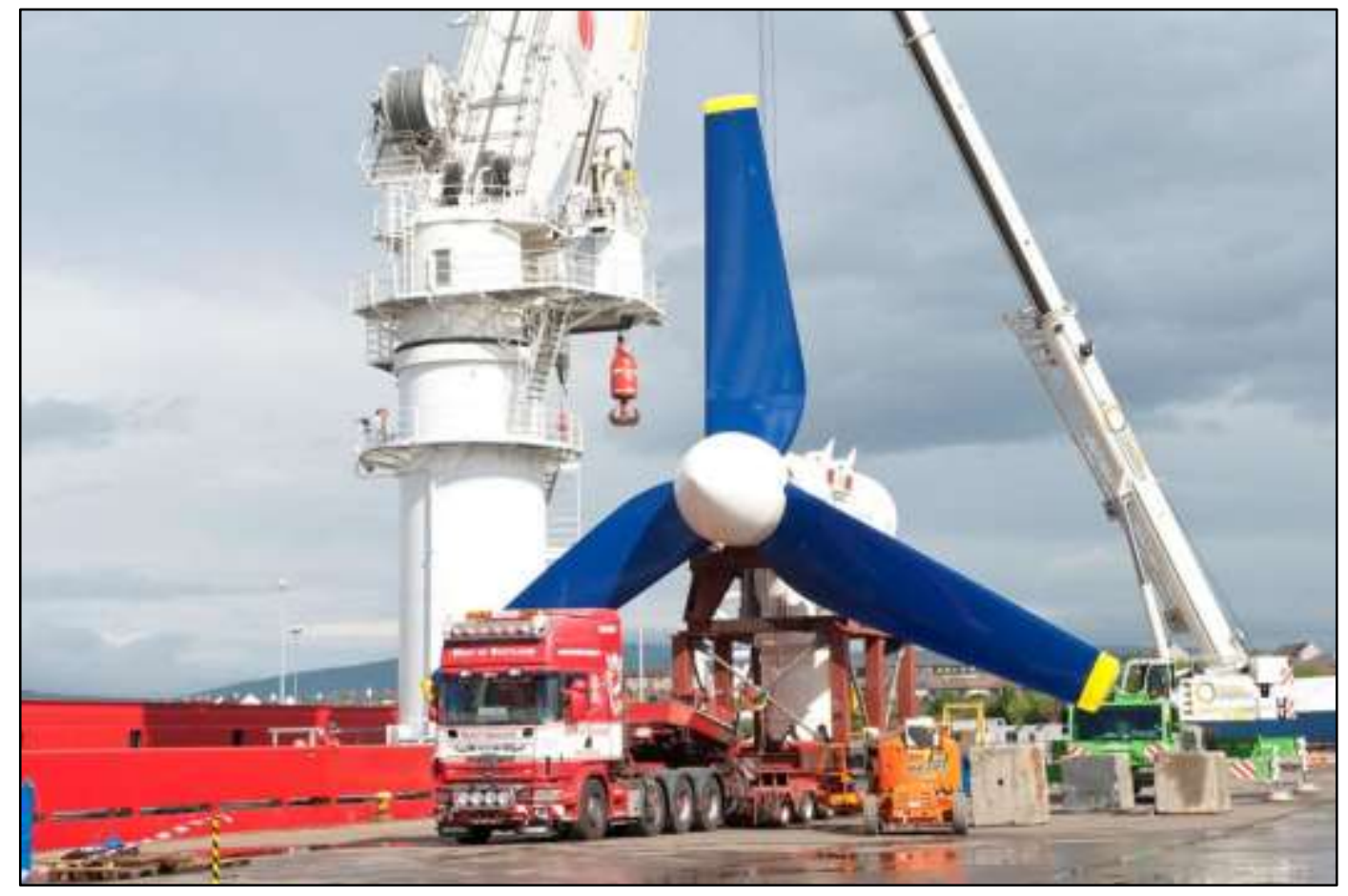

Figure 1 AR1000 built by Atlantis Resources (Atlantis, 2015)

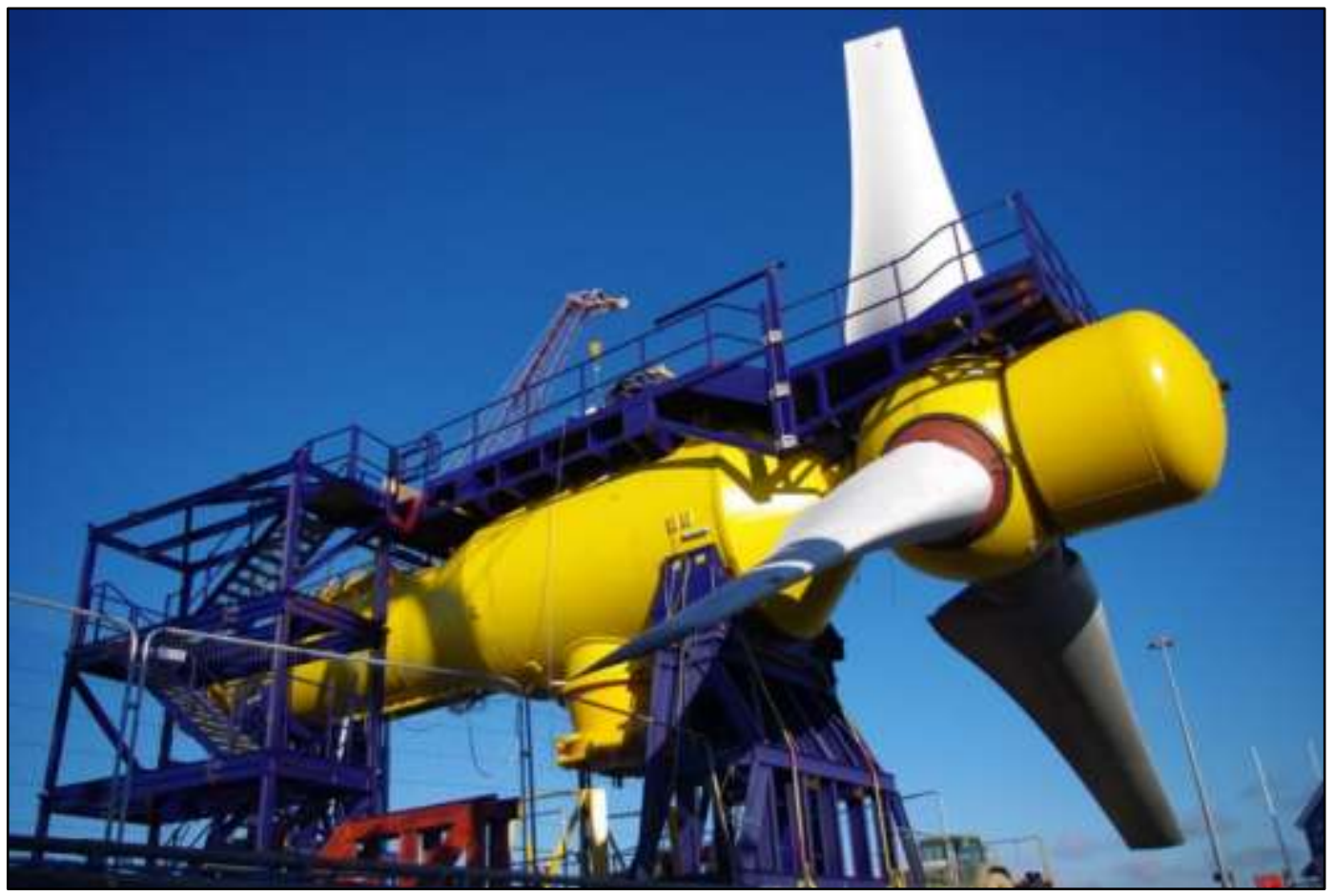

Figure 2 Tidal turbine built by Alstom (Alstom, 2013) 


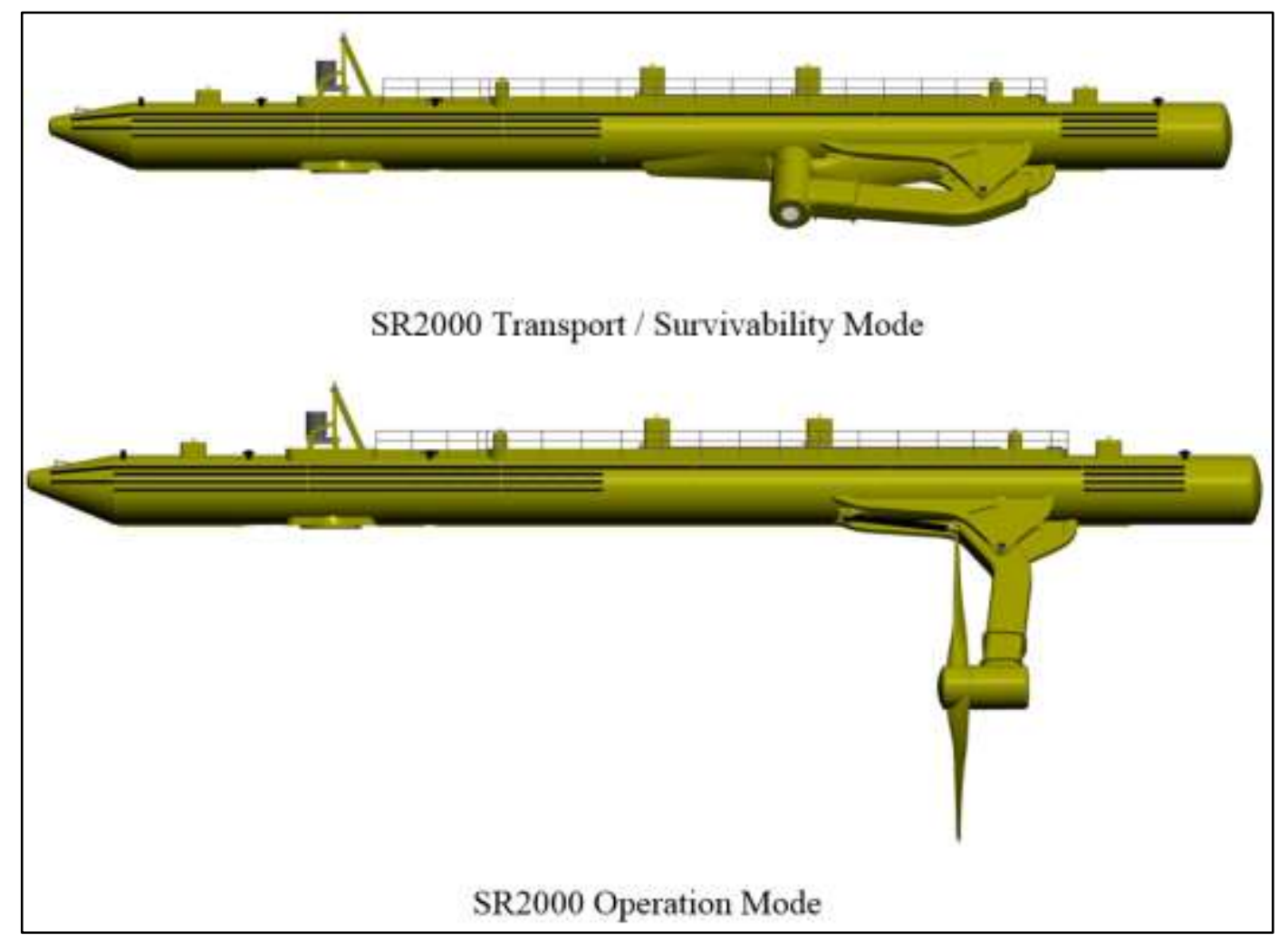


Figure 3 SR2000 2MW floating tidal energy device (Scotrenewables, 2015)

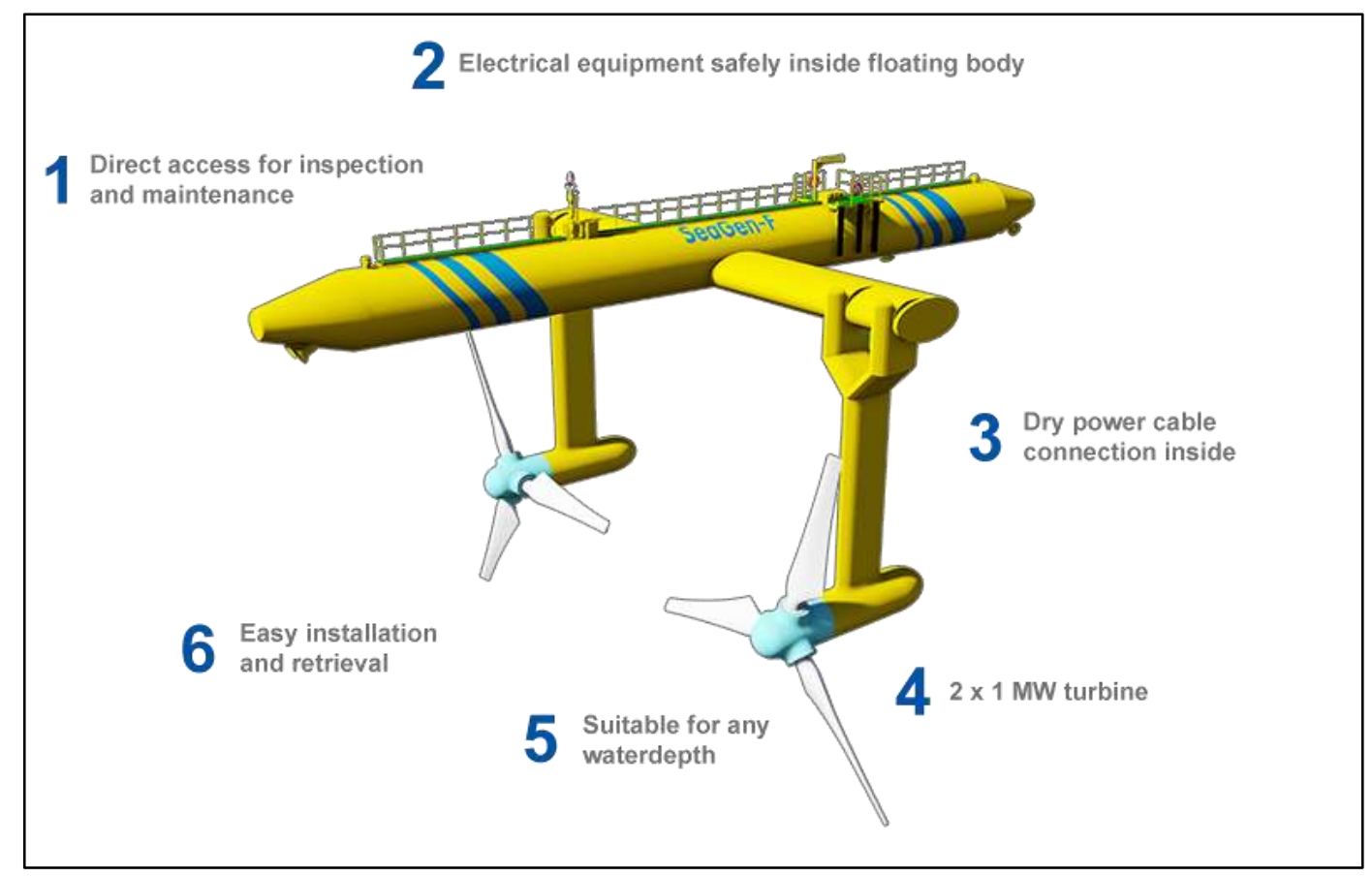

Figure 4 BlueTEC floating platform (BlueTEC, 2015)

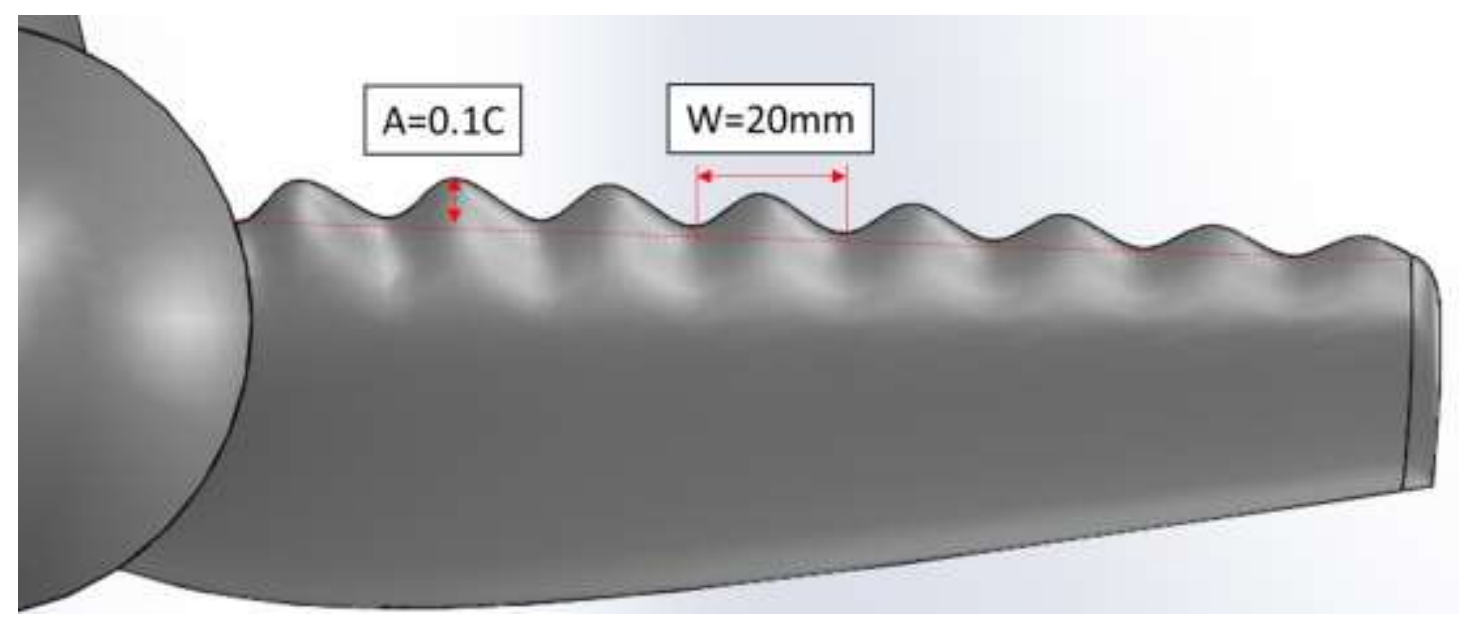

Figure 5 3D design of the turbine with leading-edge tubercles

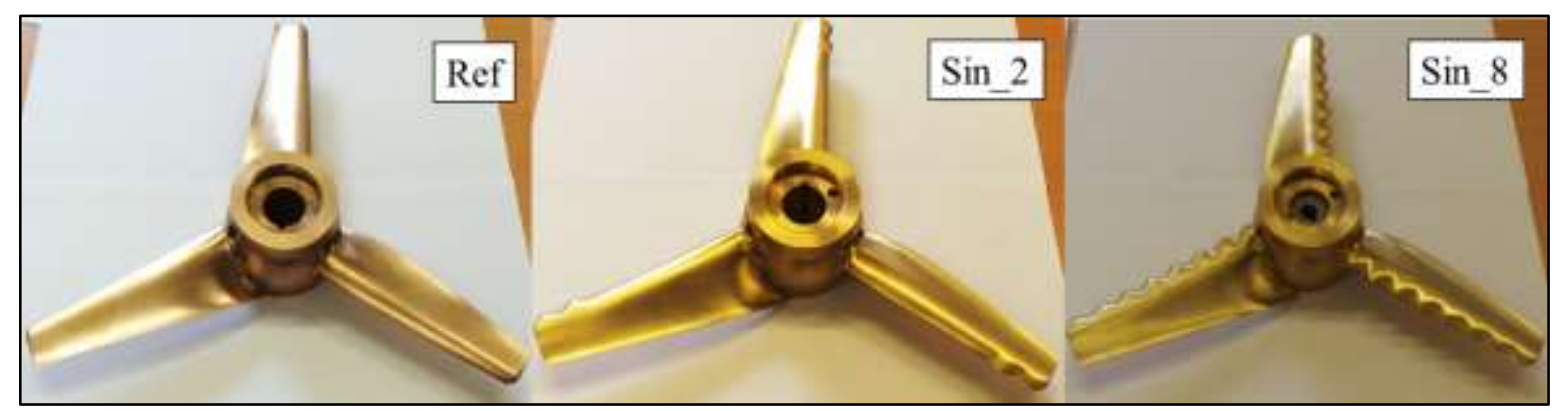

Figure 6 Tested turbine models (Left: Reference turbine with no tubercles; Middle: Turbine with partial LE tubercles; Right: Turbine with full LE Tubercles) 


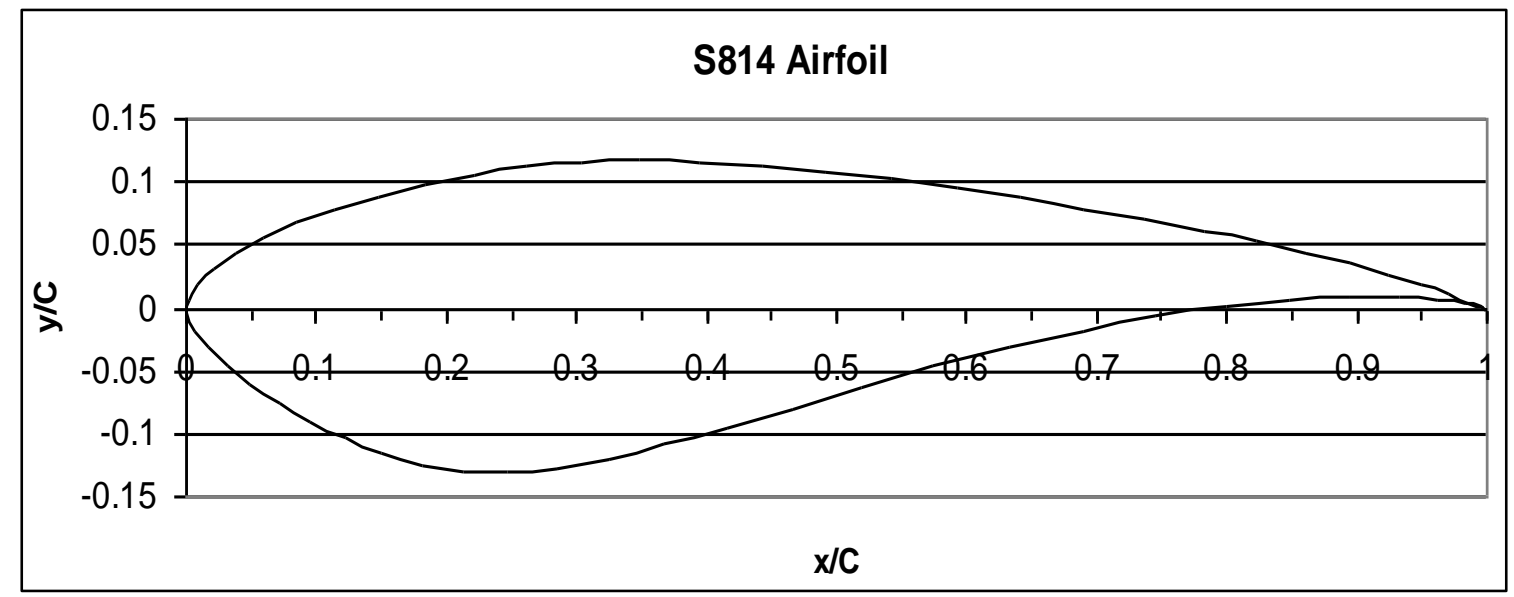

Figure 7 S814 section profile

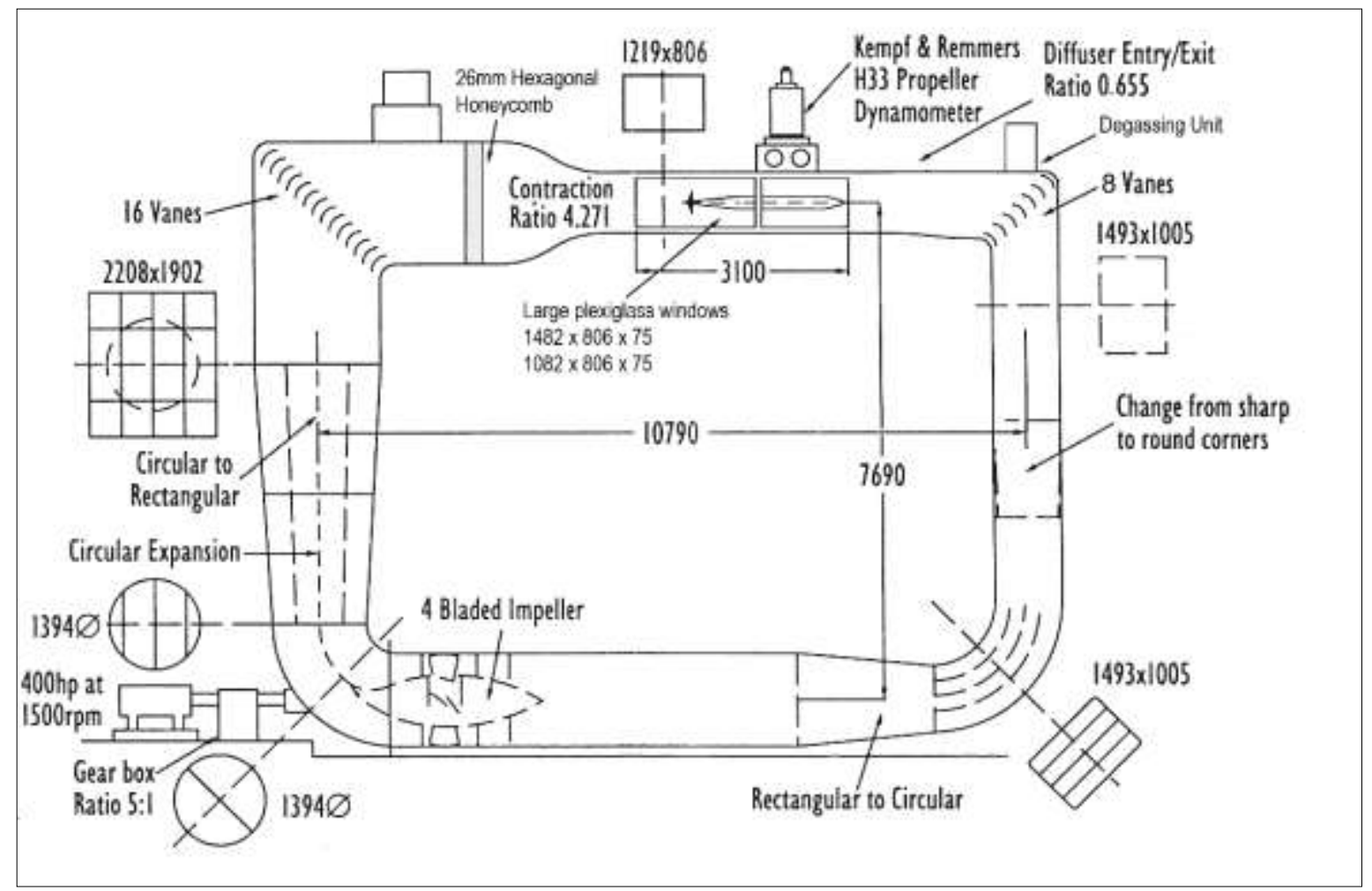

Figure 8 Sketch of ECT 


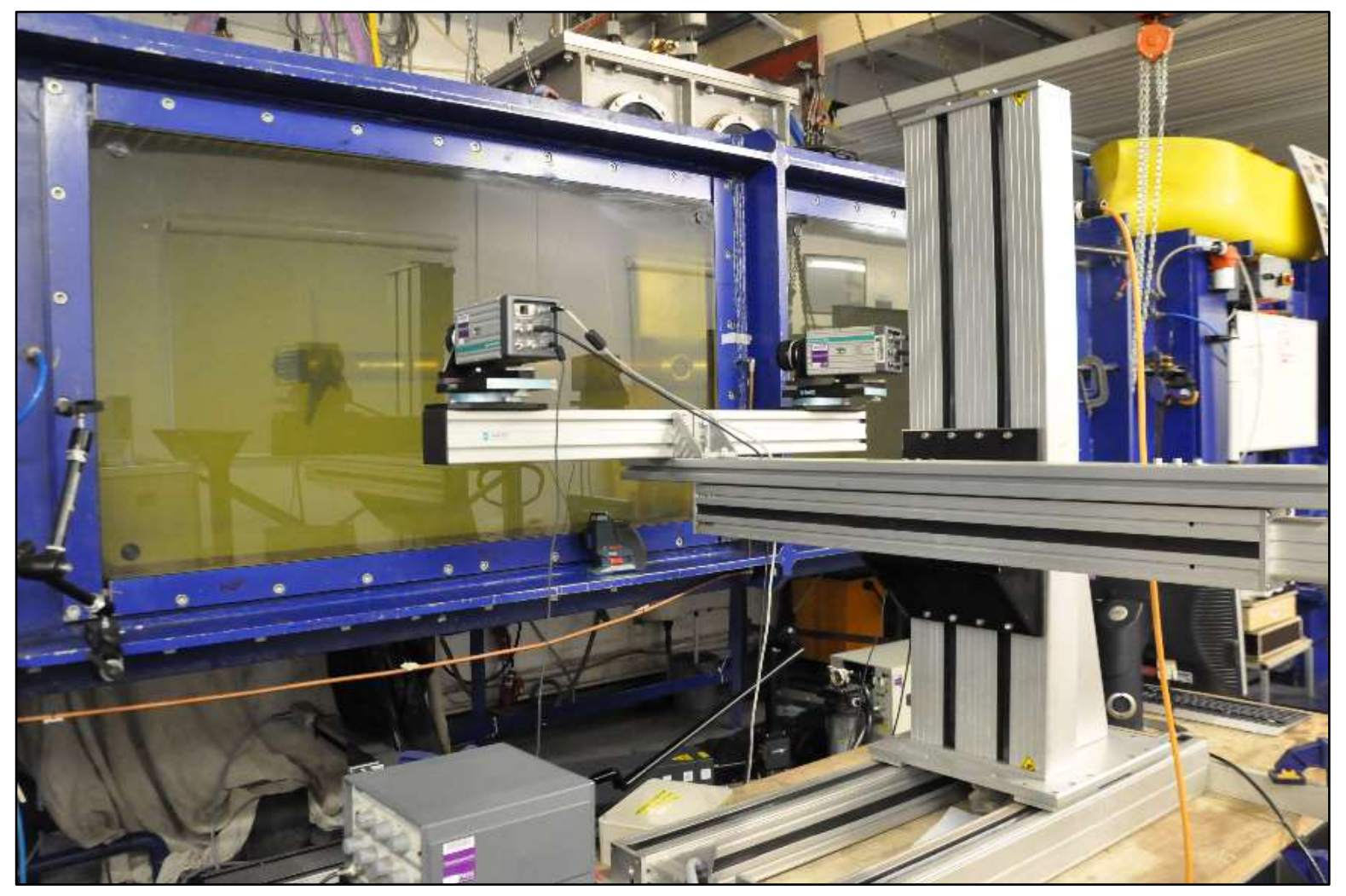

Figure 9 High-speed cameras to observe any cavitation.

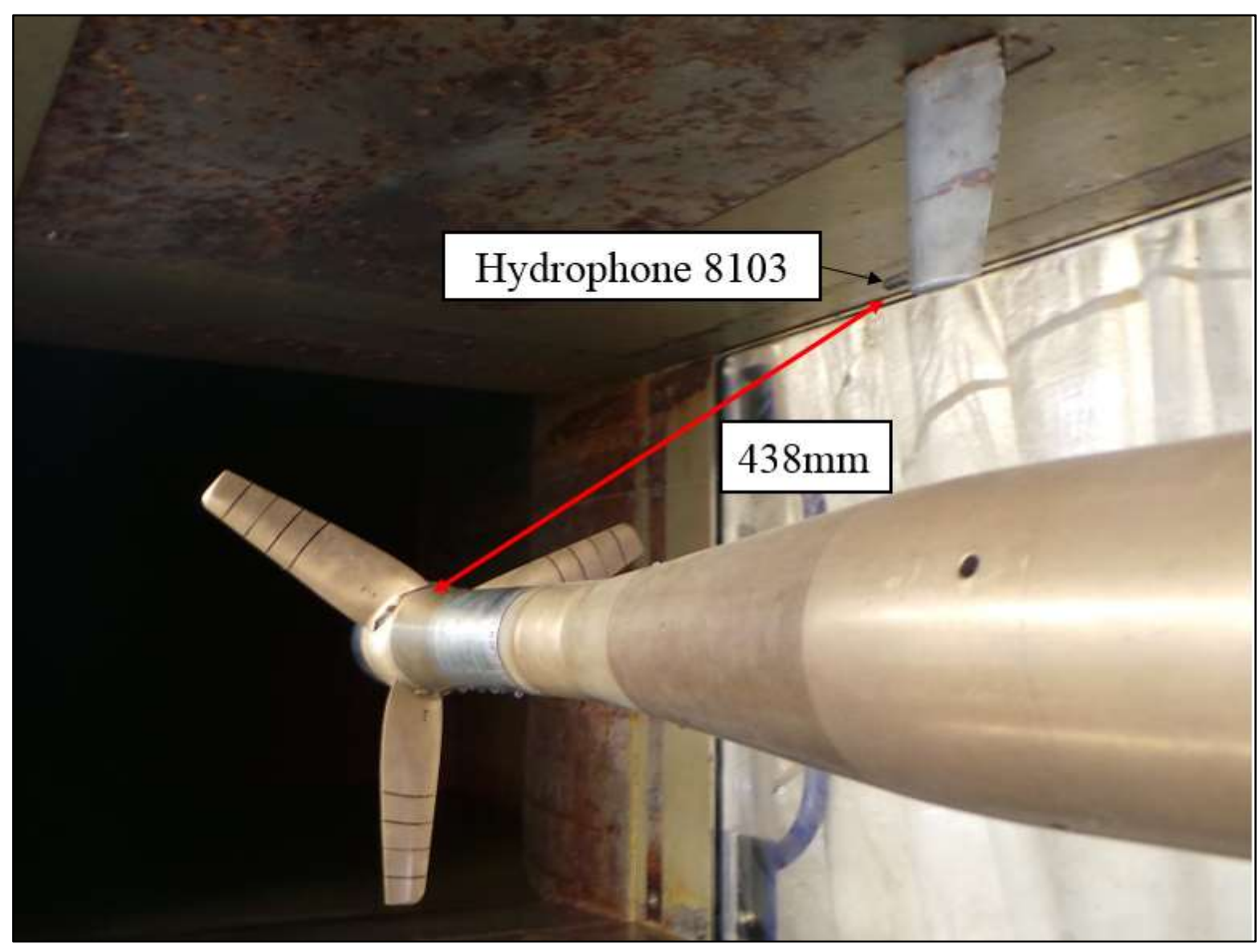

Figure 10 Arrangement of the test turbine and hydrophone 


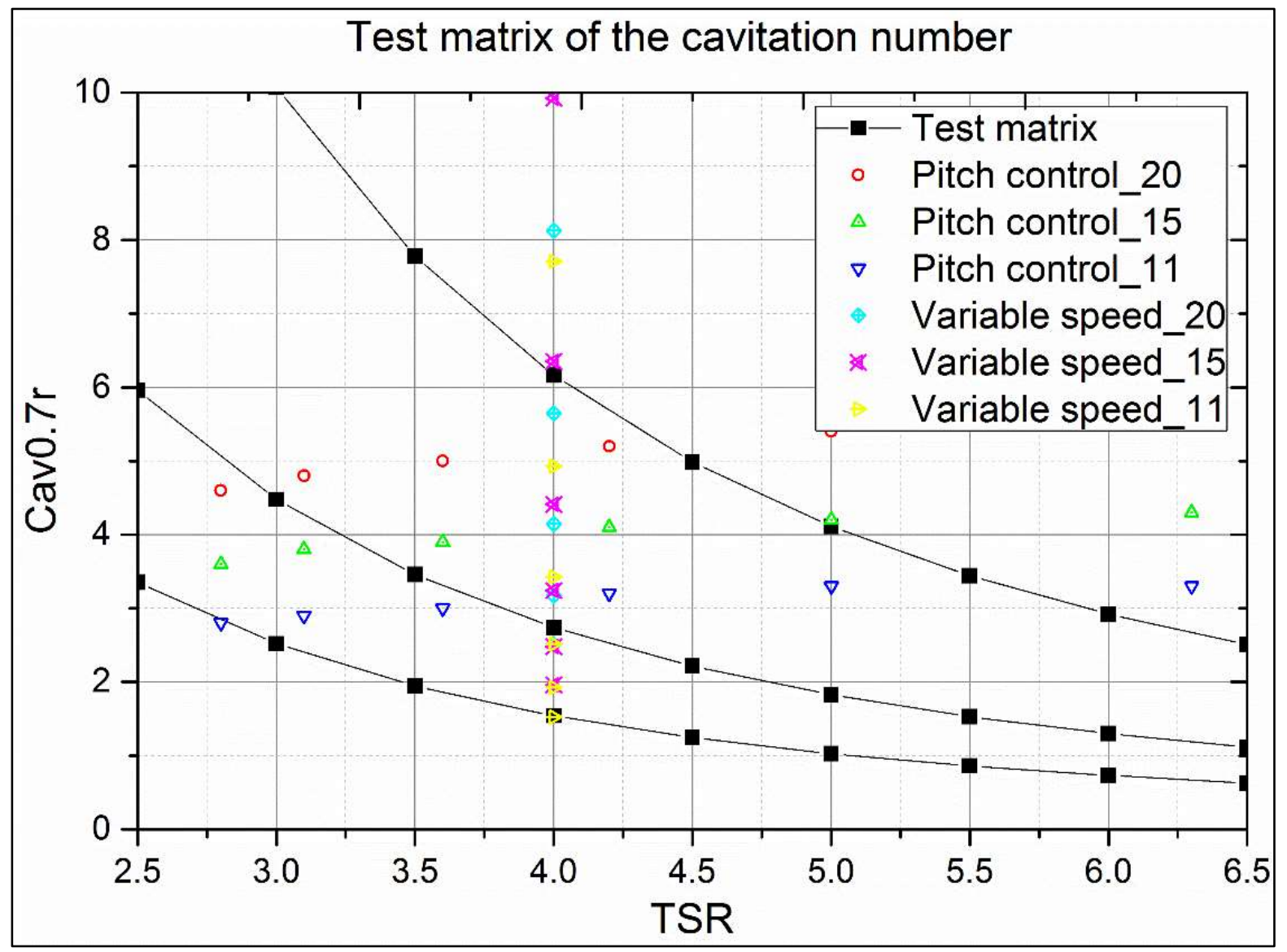

Figure 11 Test matrix against the full-scale conditions
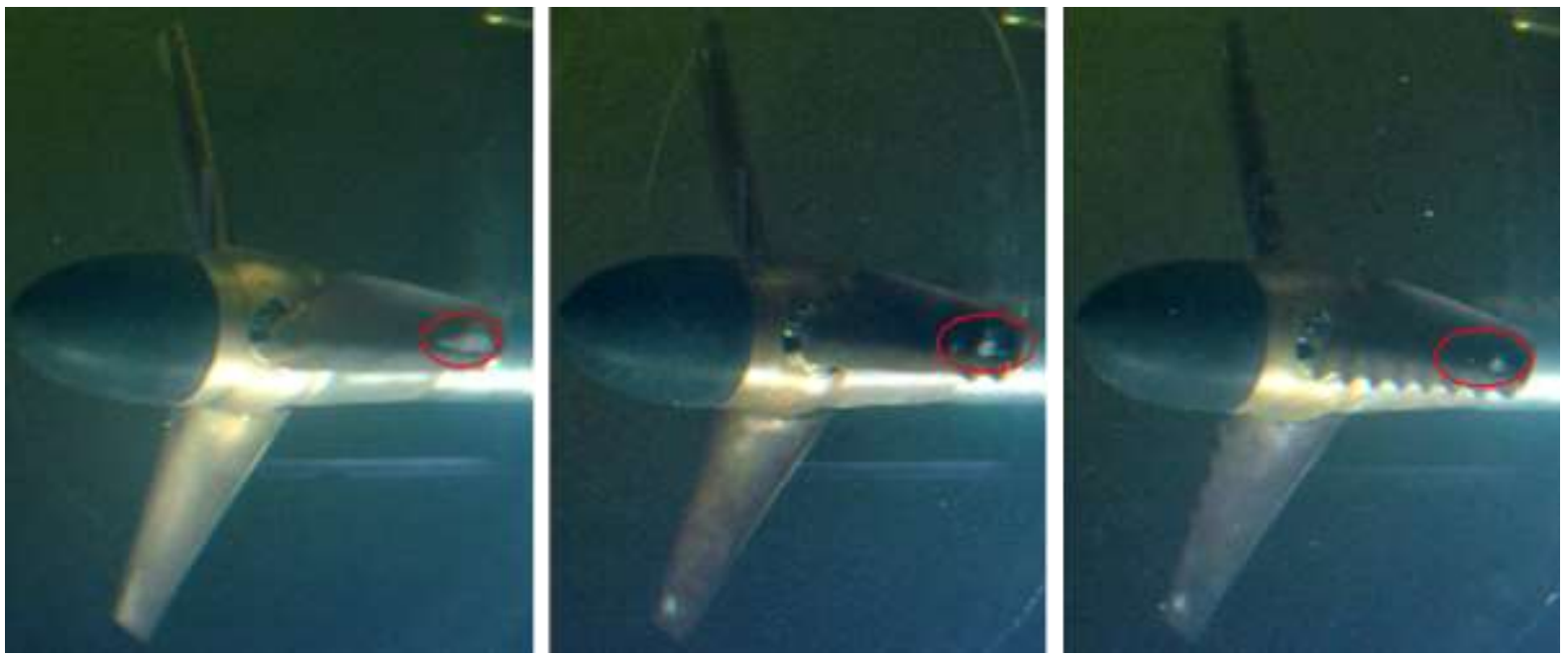

Figure 12 Detailed comparison of cavitation pattern $\left(\right.$ Pitch angle $\left.=+8^{\circ} ; \mathrm{V}=3 \mathrm{~m} / \mathrm{s} ; \mathrm{TSR}=6 ; \mathrm{Cav}_{0.75 \mathrm{r}}=1.3\right)$ 


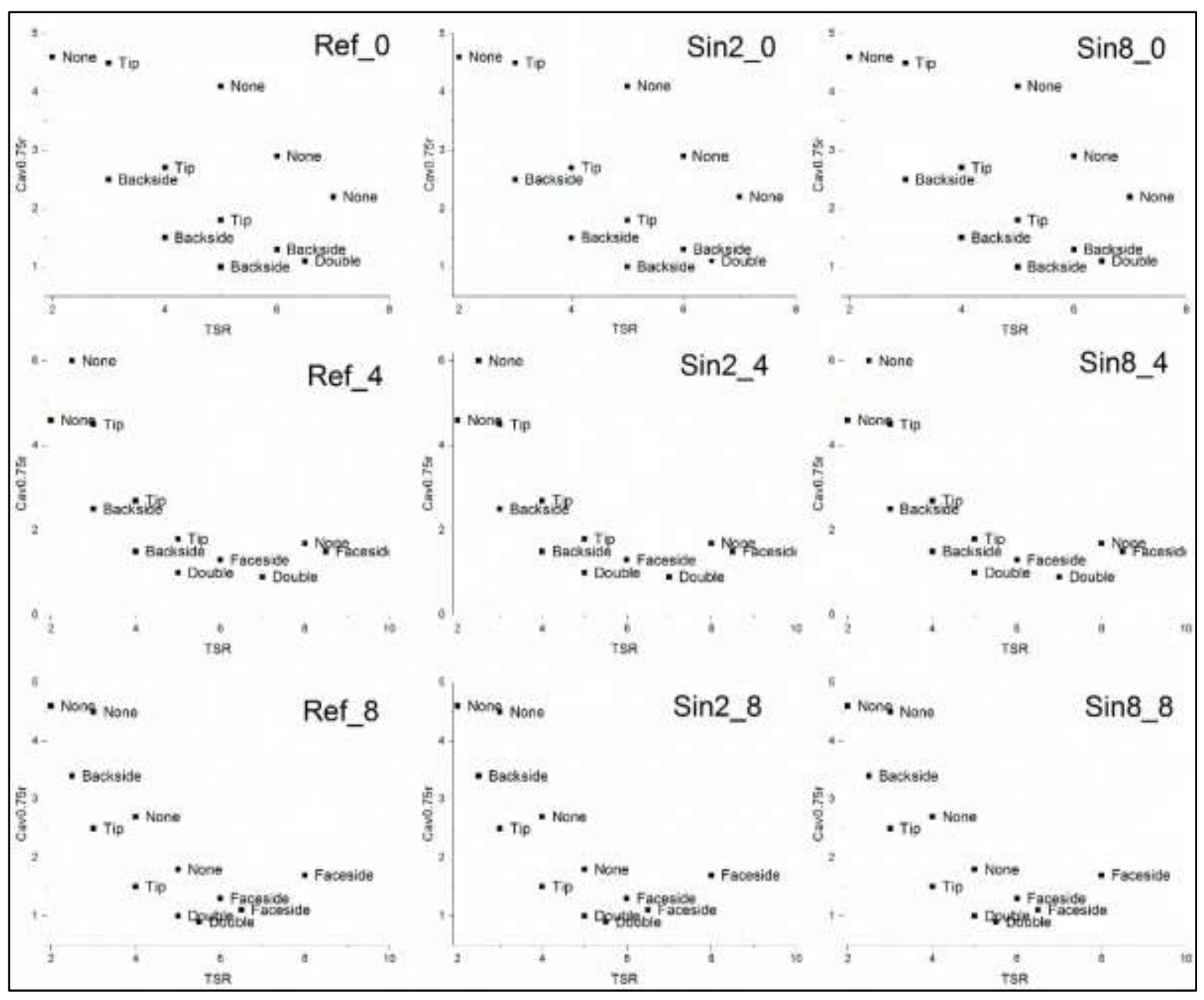

Figure 13 Cavitation inception diagram 


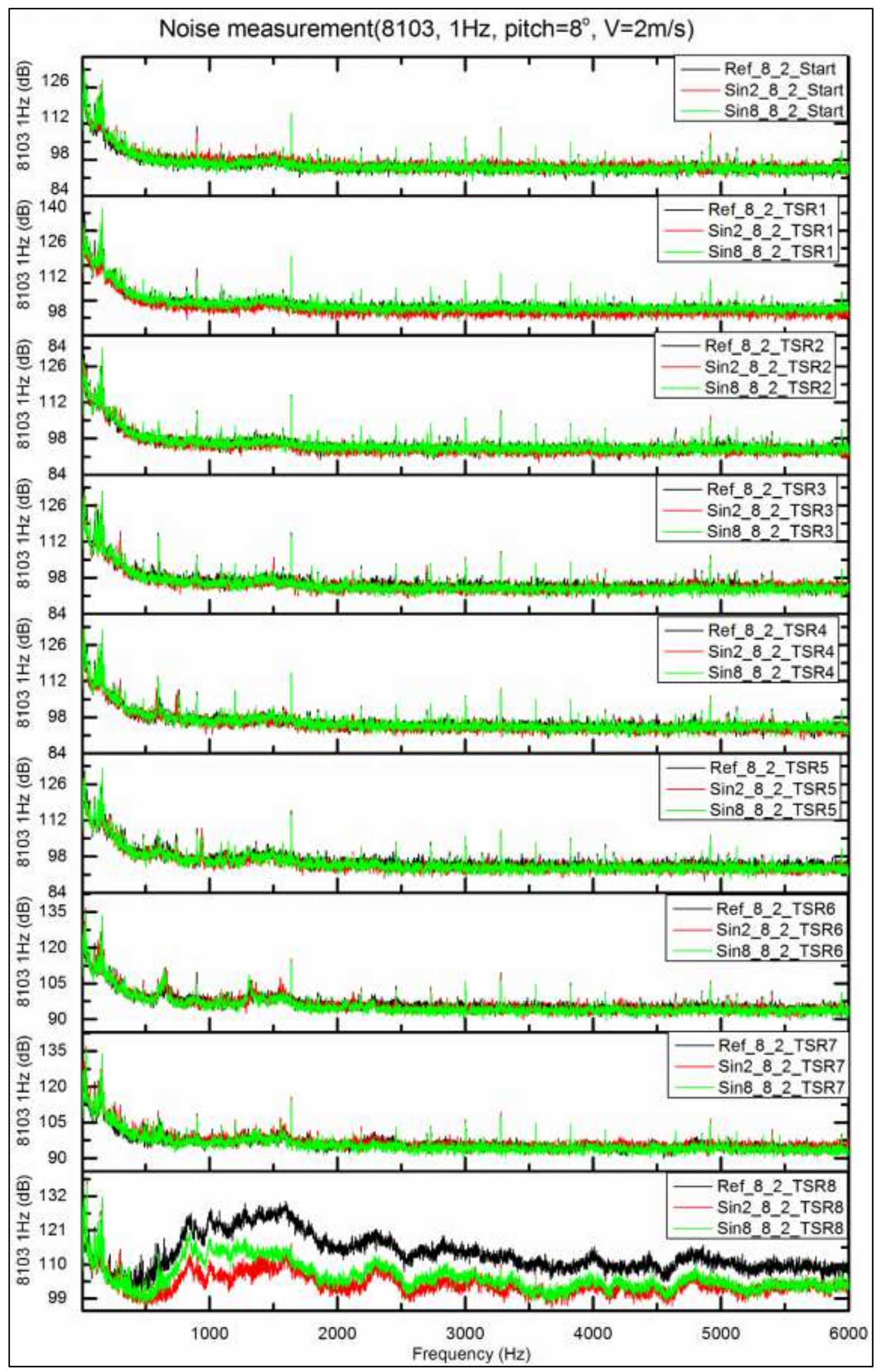

Figure 14 Comparative total noise levels measured in $1 \mathrm{~Hz}$ band (Pitch angle $=8^{\circ}, \mathrm{V}=2 \mathrm{~m} / \mathrm{s}$ ) 


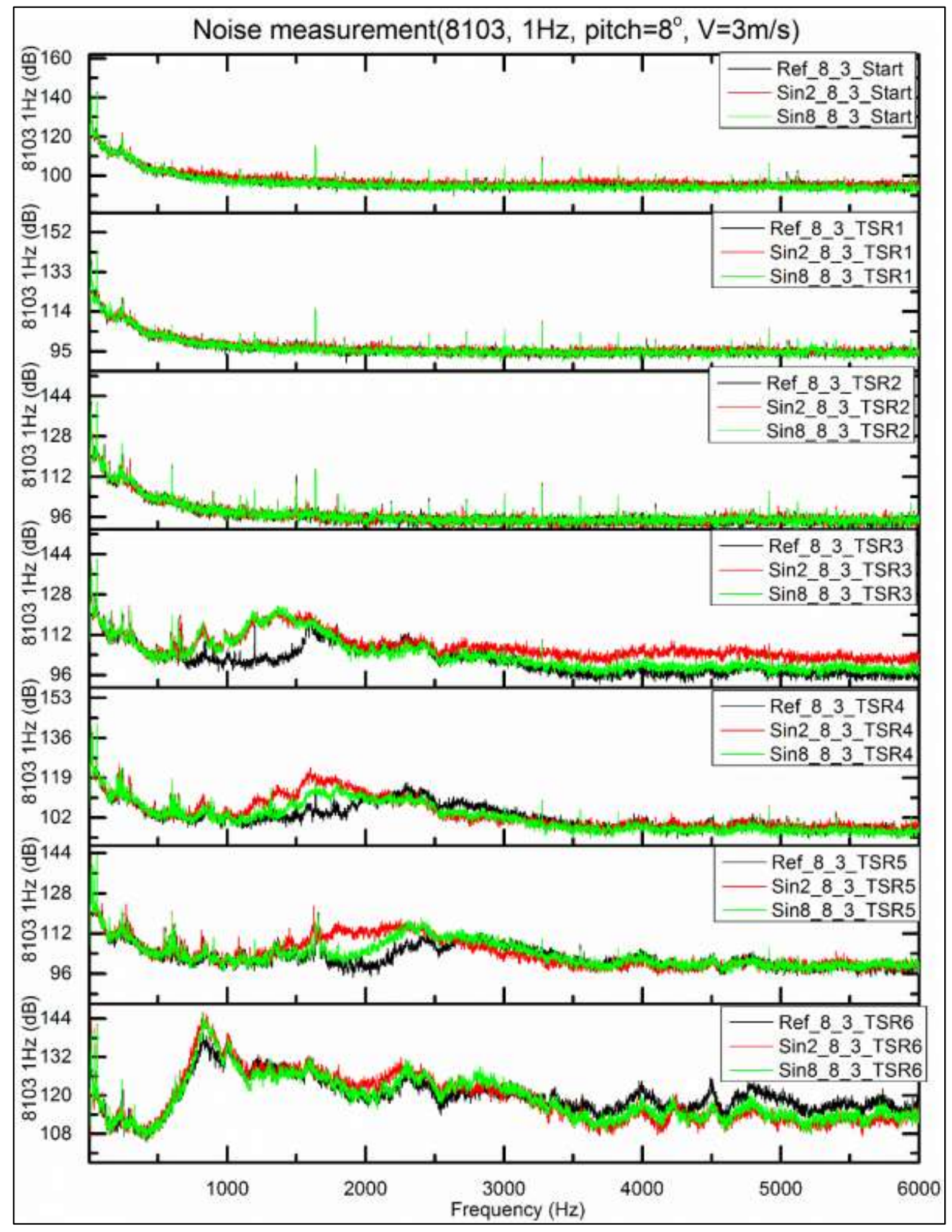

Figure 15 Comparative total noise levels measured in $1 \mathrm{~Hz}$ band (Pitch angle $=8^{\circ}, \mathrm{V}=3 \mathrm{~m} / \mathrm{s}$ ) 


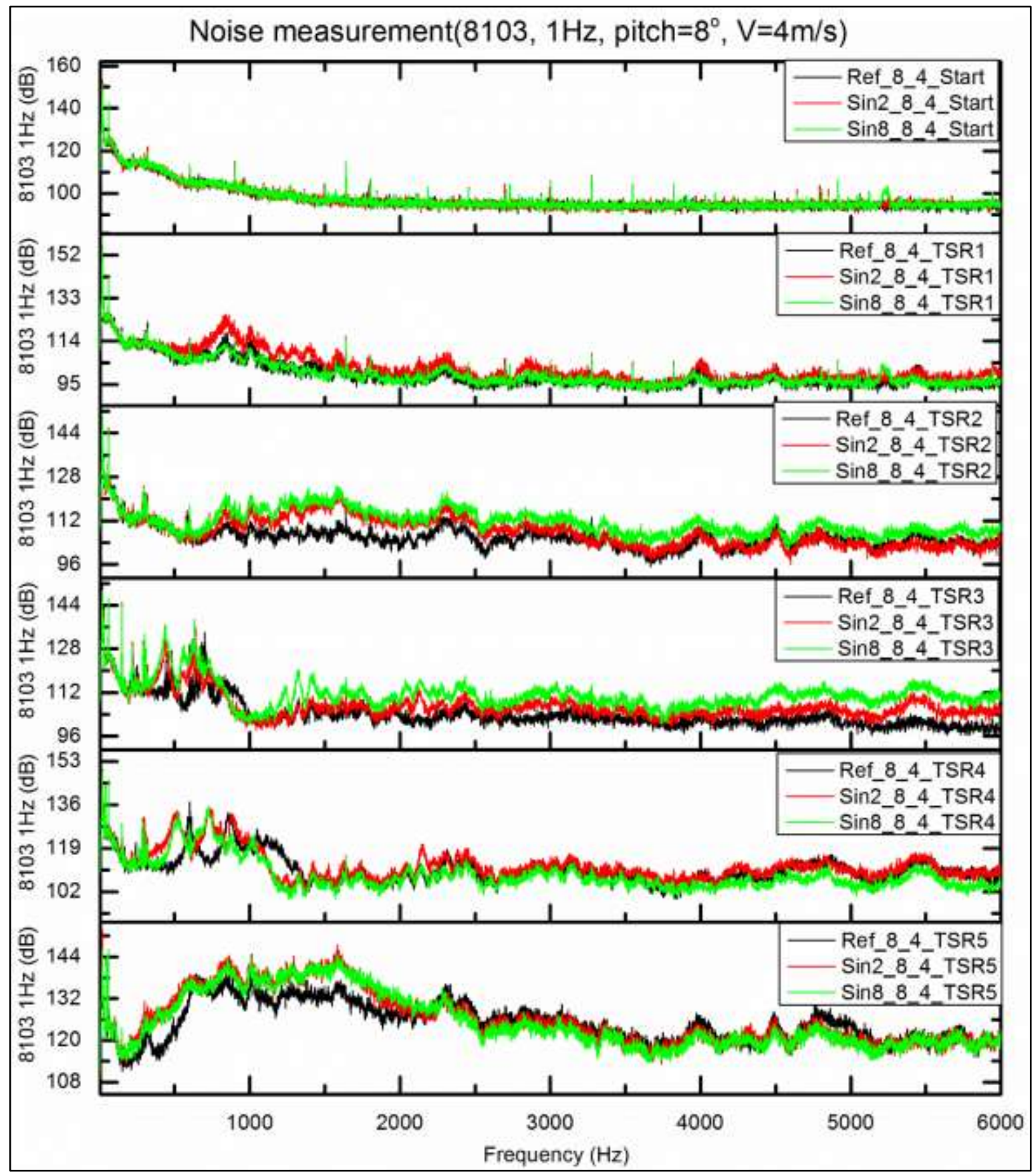

Figure 16 Comparative total noise levels measured in $1 \mathrm{~Hz}$ band (Pitch angle $=8^{\circ}, \mathrm{V}=4 \mathrm{~m} / \mathrm{s}$ ) 

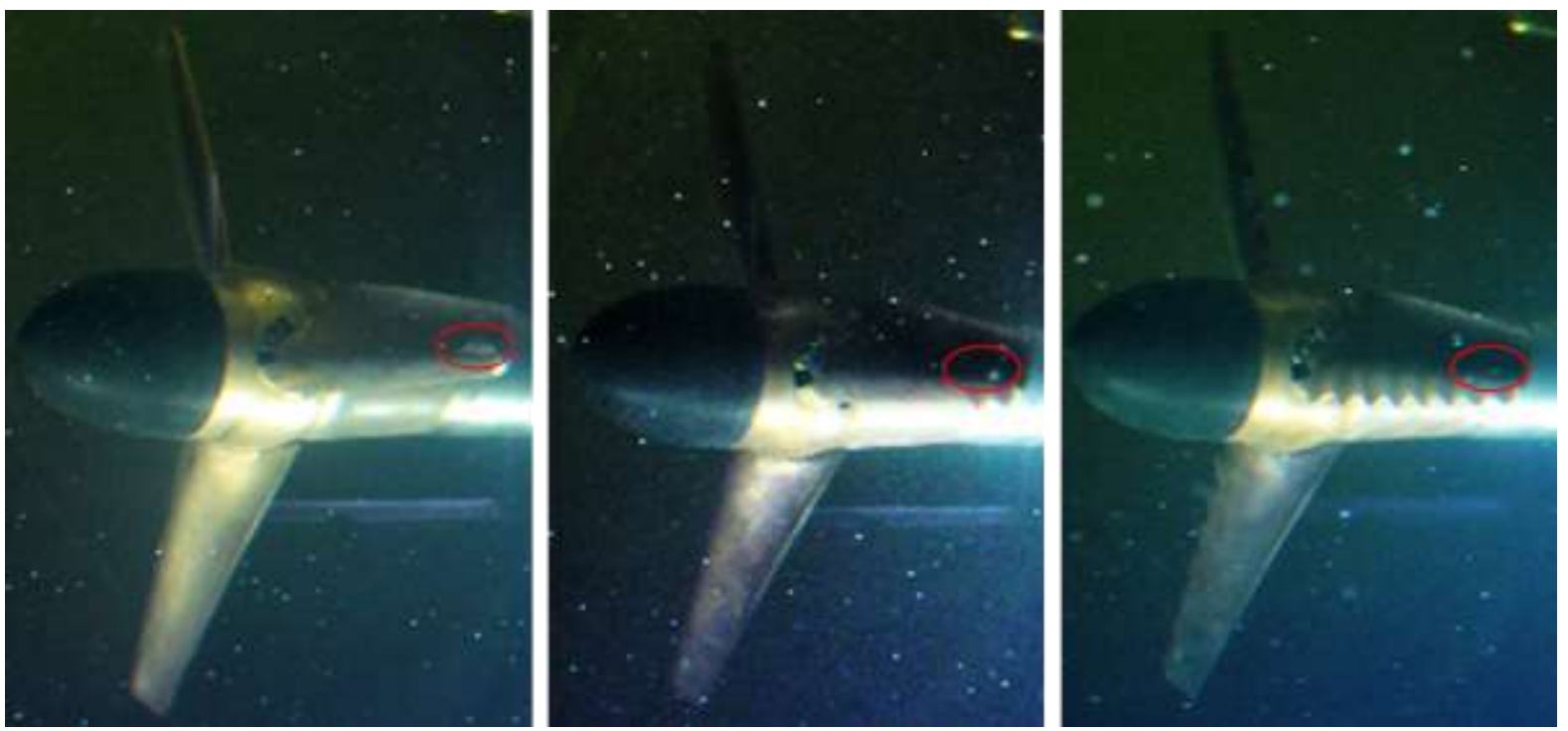

Figure 17 Detailed comparison of cavitation pattern (Pitch angle $=+8^{\circ} ; \mathrm{V}=2 \mathrm{~m} / \mathrm{s} ; \mathrm{TSR}=8$; Cav0.75r $=1.7$ )

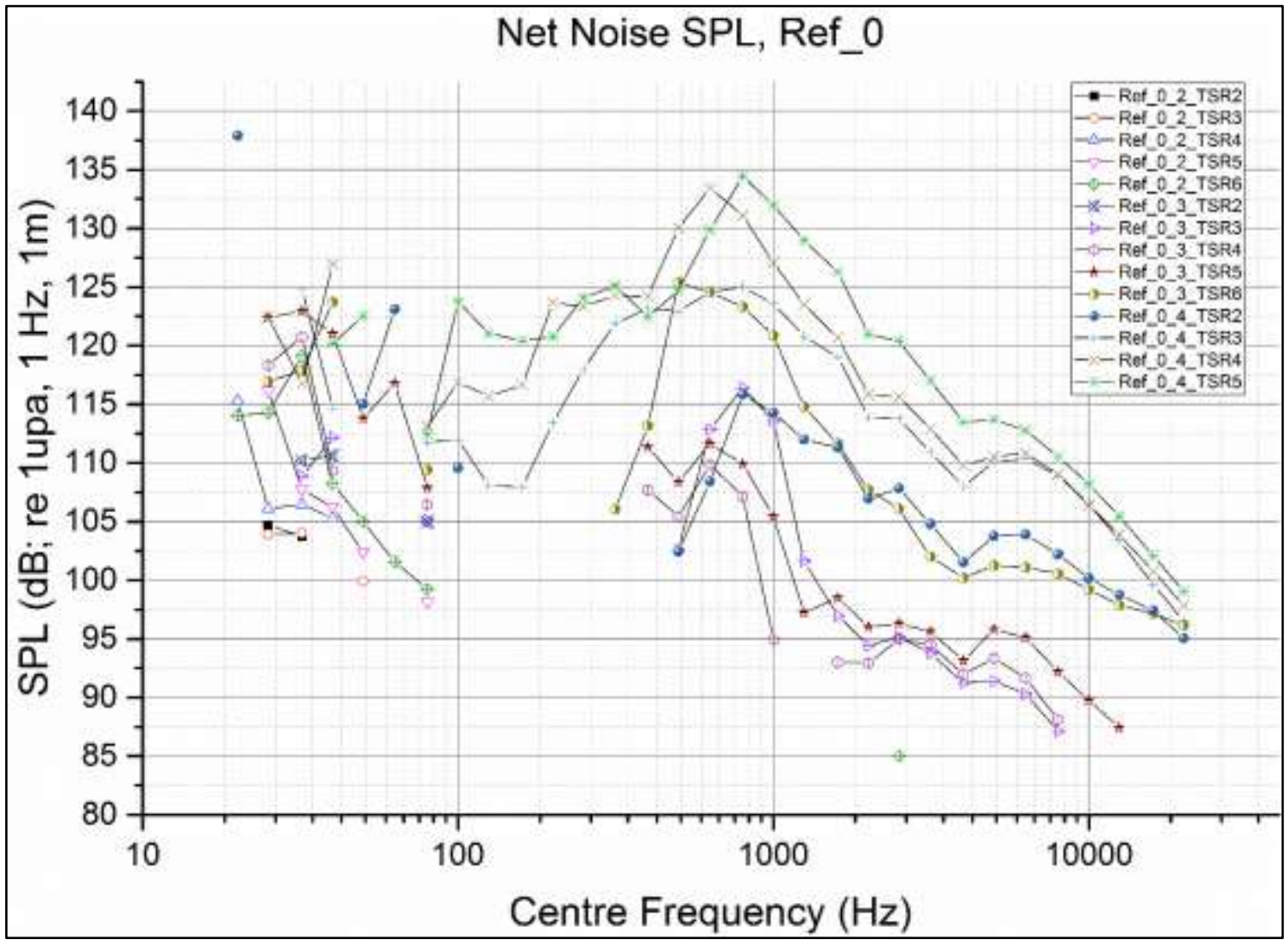

Figure 18 Net noise (sound pressure) level of Ref at pitch angle $=0^{\circ}$ 


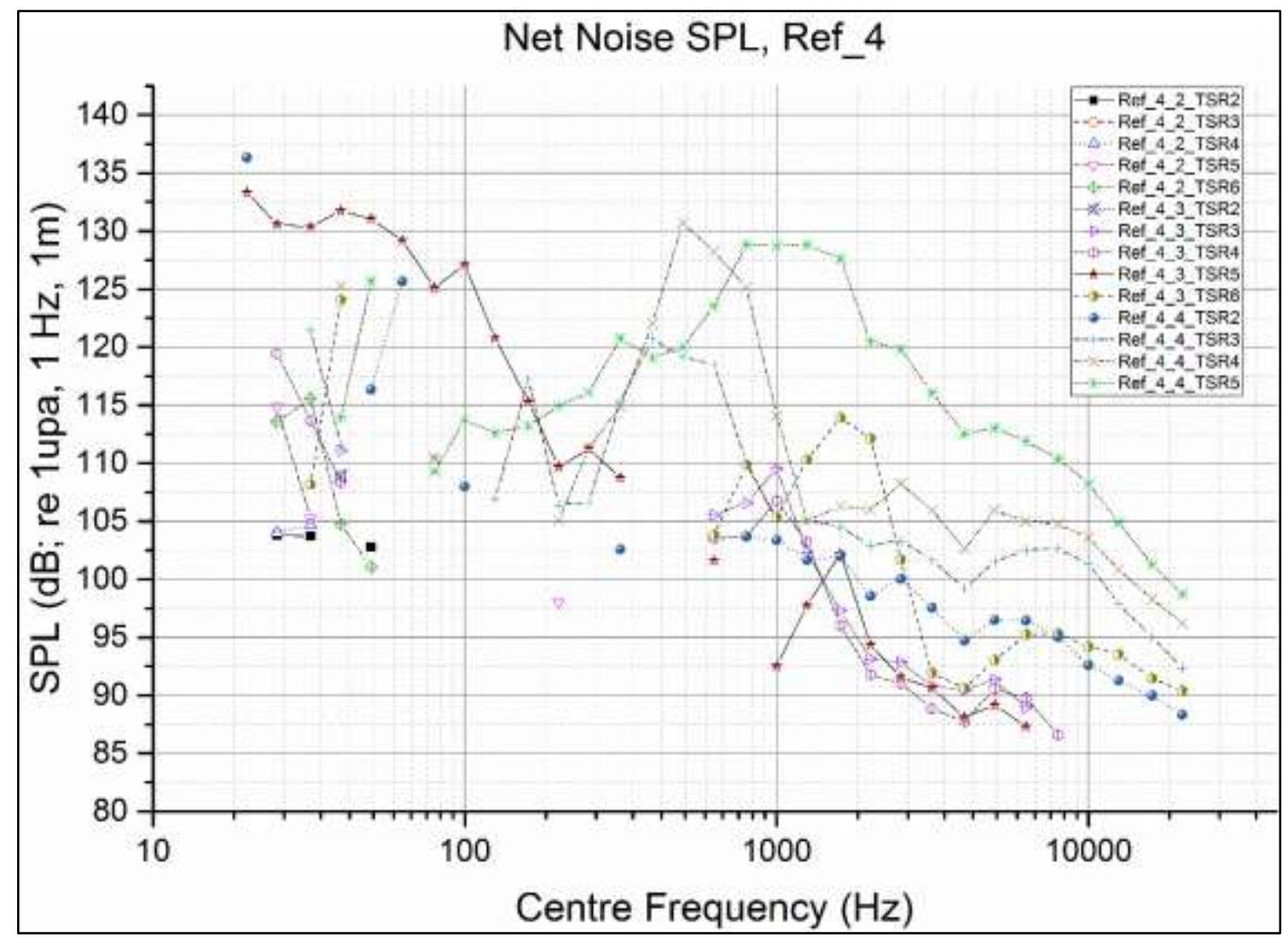

Figure 19 Net noise (sound pressure) level of Ref at pitch angle $=4^{\circ}$

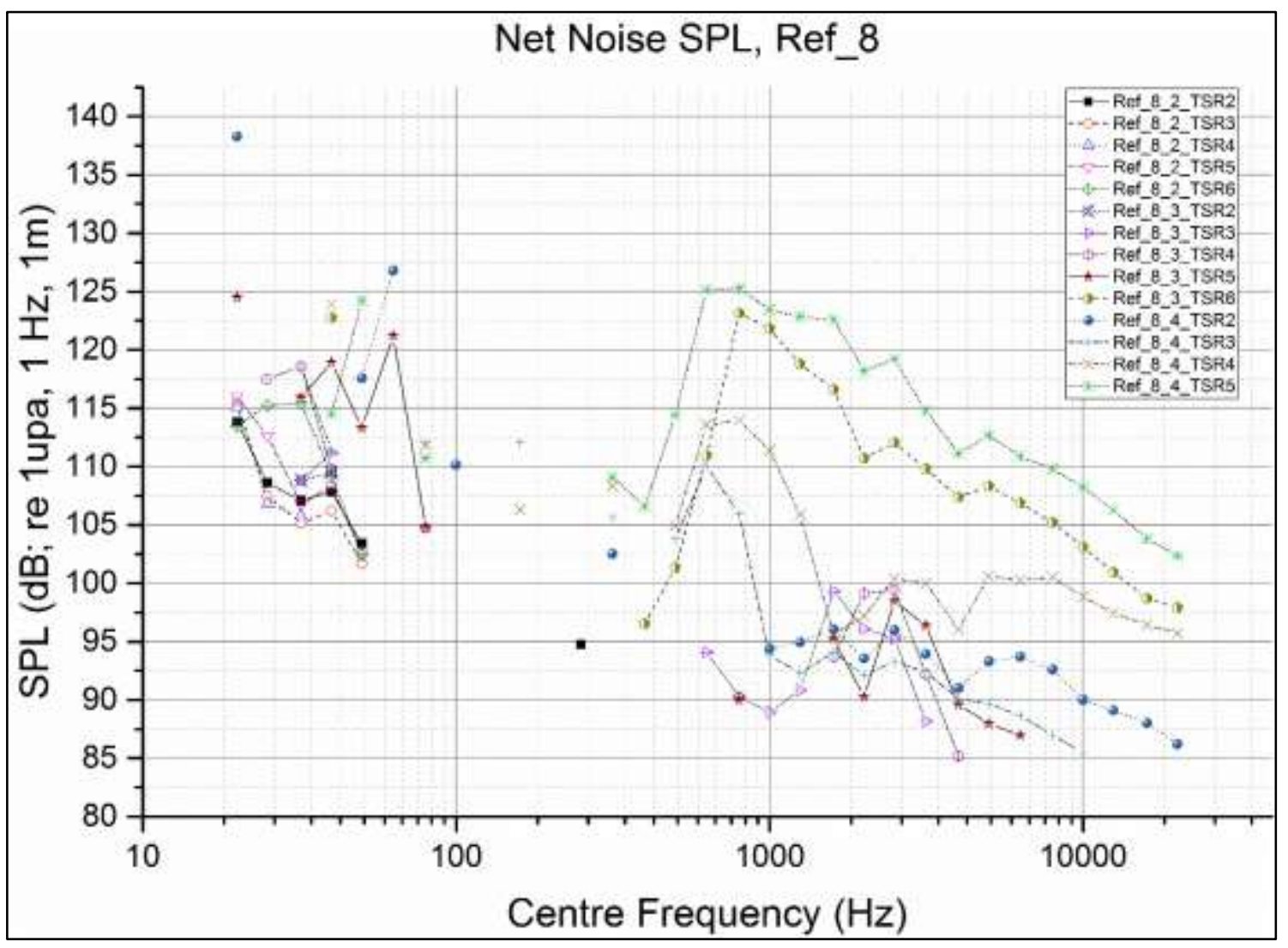

Figure 20 Net noise (sound pressure) level of Ref at pitch angle $=8^{0}$ 


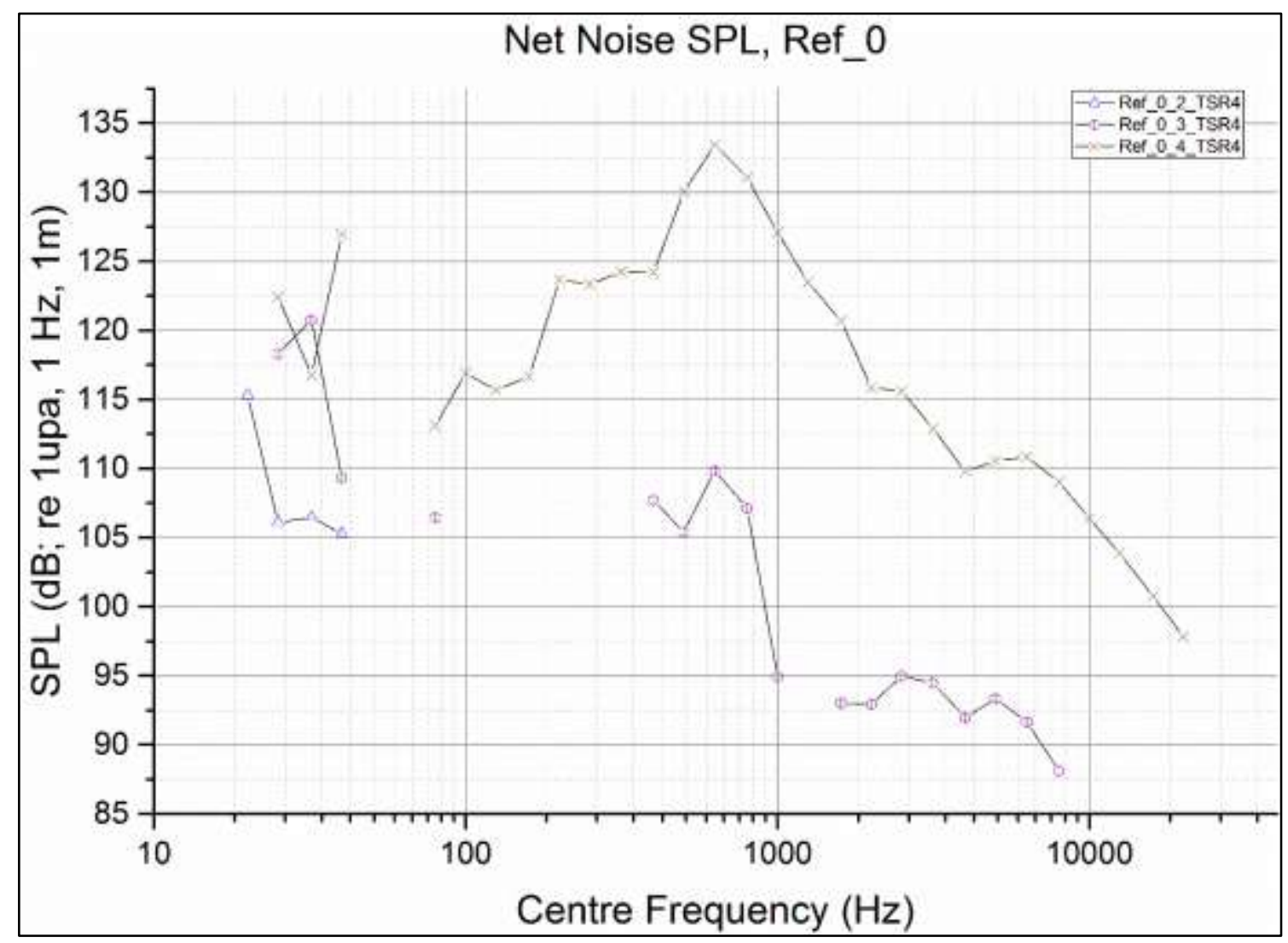

Figure 21 Net noise (sound pressure) level of Ref at pitch angle $=0^{\circ}$ and $T S R=4$

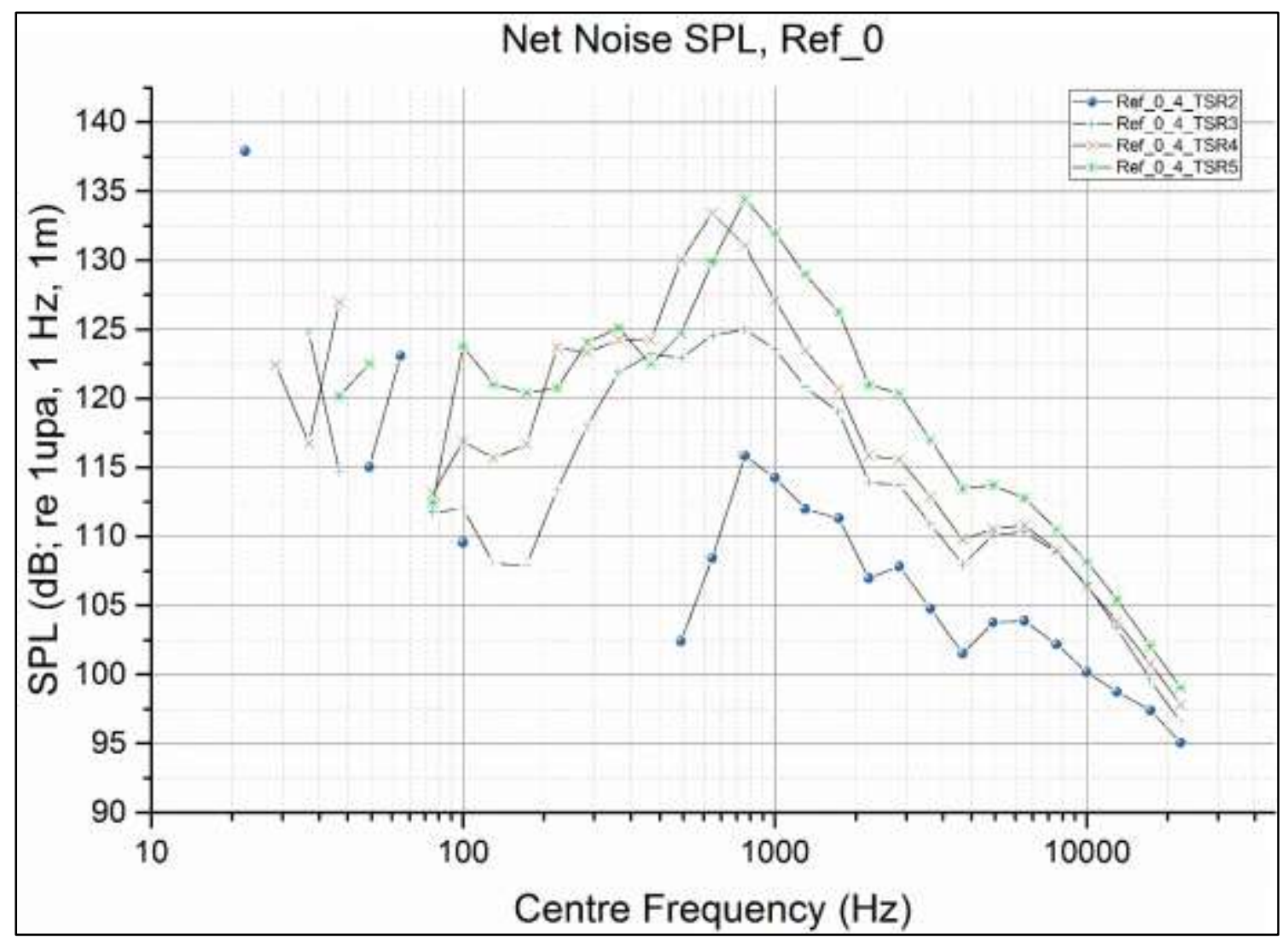

Figure 22 Net noise (sound pressure) level of Ref at pitch angle $=0^{\circ}$ and Vin $=4 \mathrm{~m} / \mathrm{s}$ 


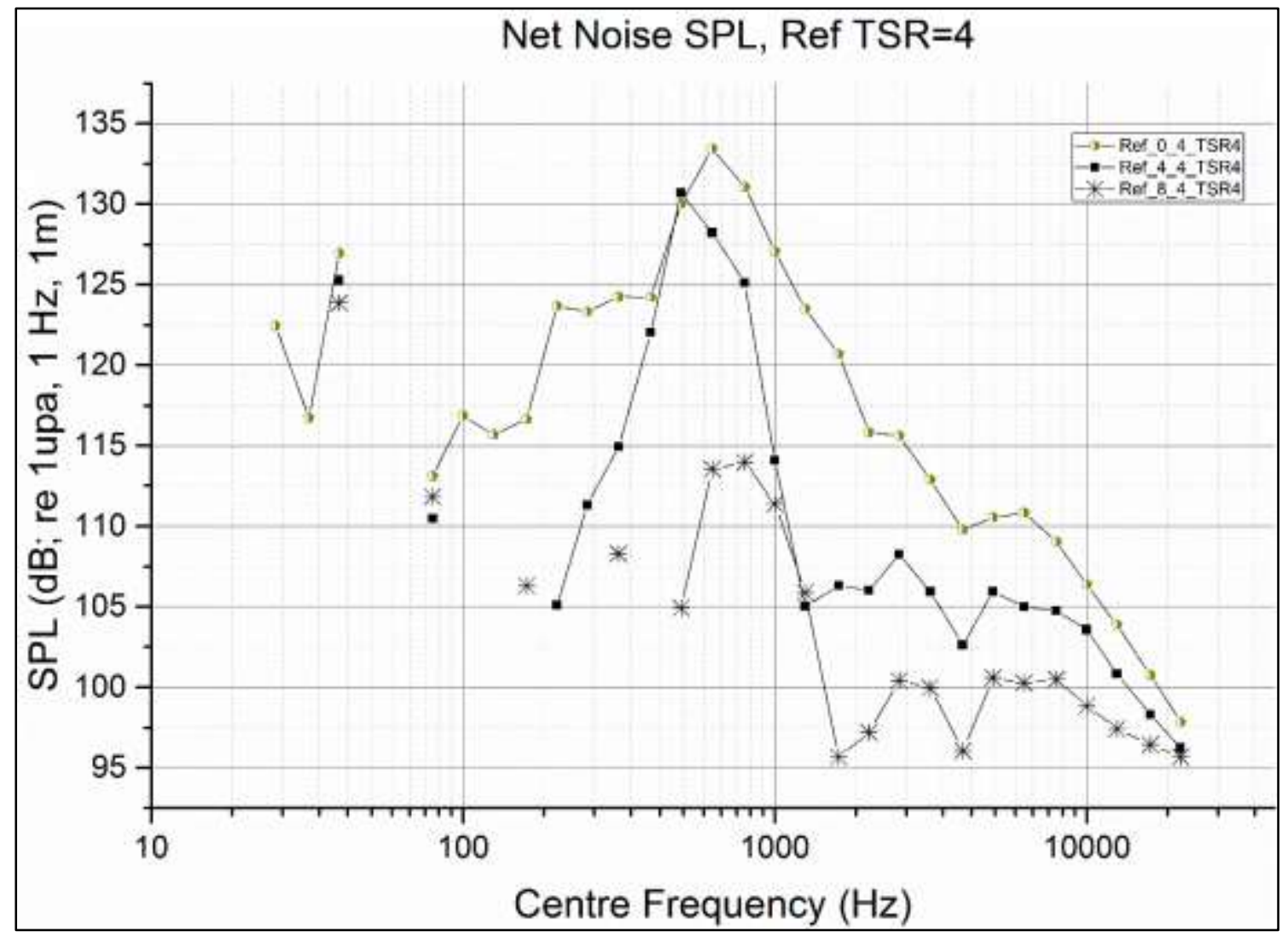

Figure 23 Net noise (sound pressure) level of Ref at TSR $=4$ and Vin $=4 \mathrm{~m} / \mathrm{s}$ 
Table 1 Full-scale design

\begin{tabular}{|c|c|c|c|}
\hline Diameter $(\mathrm{m})$ & 20 & Rotation rate $(\mathrm{RPM})$ & 12 \\
\hline Number of blades & 3 & Current speed $(\mathrm{m} / \mathrm{s})$ & 3.2 \\
\hline Immersion of shaft $(\mathrm{m})$ & 20 & Max. wave height $(\mathrm{m})$ & 9 \\
\hline
\end{tabular}

Table 2 Full-scale operating conditions for pitch control turbine

\begin{tabular}{|c|c|c|c|c|c|}
\hline \multicolumn{7}{|c|}{ Pitch control turbine with constant rotational speed } \\
\hline $\begin{array}{c}\text { Shaft } \\
\text { immersion }\end{array}$ & Diameter & Vin & RPM & TSR & Cav \\
\hline$(\mathrm{m})$ & $(\mathrm{m})$ & $(\mathrm{m} / \mathrm{s})$ & & & $(0.7 \mathrm{R})$ \\
\hline 20 & 20 & 4.5 & 12.0 & 2.8 & 4.6 \\
\hline 20 & 20 & 4.0 & 12.0 & 3.1 & 4.8 \\
\hline 20 & 20 & 3.5 & 12.0 & 3.6 & 5.0 \\
\hline 20 & 20 & 3.0 & 12.0 & 4.2 & 5.2 \\
\hline 20 & 20 & 2.5 & 12.0 & 5.0 & 5.4 \\
\hline 20 & 20 & 2.0 & 12.0 & 6.3 & 5.5 \\
\hline 15 & 20 & 4.5 & 12.0 & 2.8 & 3.6 \\
\hline 15 & 20 & 4.0 & 12.0 & 3.1 & 3.8 \\
\hline 15 & 20 & 3.5 & 12.0 & 3.6 & 3.9 \\
\hline 15 & 20 & 3.0 & 12.0 & 4.2 & 4.1 \\
\hline 15 & 20 & 2.5 & 12.0 & 5.0 & 4.2 \\
\hline 15 & 20 & 2.0 & 12.0 & 6.3 & 4.3 \\
\hline 11 & 20 & 4.5 & 12.0 & 2.8 & 2.8 \\
\hline 11 & 20 & 4.0 & 12.0 & 3.1 & 2.9 \\
\hline 11 & 20 & 3.5 & 12.0 & 3.6 & 3.0 \\
\hline 11 & 20 & 3.0 & 12.0 & 4.2 & 3.2 \\
\hline 11 & 20 & 2.5 & 12.0 & 5.0 & 3.3 \\
\hline 11 & 20 & 2.0 & 12.0 & 6.3 & 3.3 \\
\hline
\end{tabular}

Table 3 Full-scale operating conditions for variable speed control turbine

\begin{tabular}{|c|c|c|c|c|c|}
\hline \multicolumn{7}{|c|}{ Variable speed control turbine with a constant TSR } \\
\hline $\begin{array}{c}\text { Shaft } \\
\text { immersion }\end{array}$ & Diameter & Vin & RPM & TSR & Cav \\
\hline$(\mathrm{m})$ & $(\mathrm{m})$ & $(\mathrm{m} / \mathrm{s})$ & & & $(0.7 \mathrm{R})$ \\
\hline 20 & 20 & 4.5 & 17.189 & 4.0 & 2.5 \\
\hline 20 & 20 & 4.0 & 15.279 & 4.0 & 3.2 \\
\hline 20 & 20 & 3.5 & 13.369 & 4.0 & 4.1 \\
\hline 20 & 20 & 3.0 & 11.459 & 4.0 & 5.6 \\
\hline 20 & 20 & 2.5 & 9.549 & 4.0 & 8.1 \\
\hline 20 & 20 & 2.0 & 7.639 & 4.0 & 12.7 \\
\hline 15 & 20 & 4.5 & 17.189 & 4.0 & 2.0 \\
\hline 15 & 20 & 4.0 & 15.279 & 4.0 & 2.5 \\
\hline 15 & 20 & 3.5 & 13.369 & 4.0 & 3.2 \\
\hline 15 & 20 & 3.0 & 11.459 & 4.0 & 4.4 \\
\hline 15 & 20 & 2.5 & 9.549 & 4.0 & 6.4 \\
\hline 15 & 20 & 2.0 & 7.639 & 4.0 & 9.9 \\
\hline 11 & 20 & 4.5 & 17.189 & 4.0 & 1.5 \\
\hline 11 & 20 & 4.0 & 15.279 & 4.0 & 1.9 \\
\hline 11 & 20 & 3.5 & 13.369 & 4.0 & 2.5 \\
\hline 11 & 20 & 3.0 & 11.459 & 4.0 & 3.4 \\
\hline 11 & 20 & 2.5 & 9.549 & 4.0 & 4.9 \\
\hline 11 & 20 & 2.0 & 7.639 & 4.0 & 7.7 \\
\hline
\end{tabular}

Table 4 Main particulars of the tidal stream turbine model

\begin{tabular}{|c|c|c|c|c|c|c|c|c|c|}
\hline $\mathrm{r} / \mathrm{R}$ & 0.2 & 0.3 & 0.4 & 0.5 & 0.6 & 0.7 & 0.8 & 0.9 & 1 \\
\hline Chord length $(\mathrm{mm})$ & 64.35 & 60.06 & 55.76 & 51.47 & 47.18 & 42.88 & 38.59 & 34.29 & 30 \\
\hline Pitch angle $(\mathrm{deg})$ & 27 & 15 & 7.5 & 4 & 2 & 0.5 & -0.4 & -1.3 & -2 \\
\hline
\end{tabular}


Table 5 Hydrophone data acquisition settings

\begin{tabular}{|c|c|}
\hline Lower centre frequency & $20 \mathrm{~Hz}$ \\
\hline Upper centre frequency & $20 \mathrm{kHz}$ \\
\hline Acoustic weighting & as signal \\
\hline Reference Pressure & $1 \mu \mathrm{Pa}$ \\
\hline Overall bands - weighting & none \\
\hline
\end{tabular}

Table 6 Test matrix

\begin{tabular}{|c|c|c|c|c|c|}
\hline $\mathrm{V}$ & TSR & RPM & Pitch angle & Tunnel pressure & Cav \\
\hline$(\mathrm{m} / \mathrm{s})$ & & & $\left(^{\circ}\right)$ & $(\mathrm{mmhg})$ & $(0.7 \mathrm{r})$ \\
\hline 2 & $0.5 \sim 8$ & $47 \sim 763$ & 0 & 850 & $48.534 \sim 1.684$ \\
\hline 2 & $0.5 \sim 8$ & $47 \sim 763$ & +4 & 850 & $48.534 \sim 1.684$ \\
\hline 2 & $0.5 \sim 8$ & $47 \sim 763$ & +8 & 850 & $48.534 \sim 1.684$ \\
\hline 3 & $0.5 \sim 8$ & $71 \sim 1145$ & 0 & 850 & $21.571 \sim 0.748$ \\
\hline 3 & $0.5 \sim 8$ & $71 \sim 1145$ & +4 & 850 & $21.571 \sim 0.748$ \\
\hline 3 & $0.5 \sim 8$ & $71 \sim 1145$ & +8 & 850 & $21.571 \sim 0.748$ \\
\hline 4 & $0.5 \sim 8$ & $95 \sim 1527$ & 0 & 850 & $12.134 \sim 0.421$ \\
\hline 4 & $0.5 \sim 8$ & $95 \sim 1527$ & +4 & 850 & $12.134 \sim 0.421$ \\
\hline 4 & $0.5 \sim 8$ & $95 \sim 1527$ & +8 & 850 & $12.134 \sim 0.421$ \\
\hline
\end{tabular}


Table 7 Tip vortex cavitation

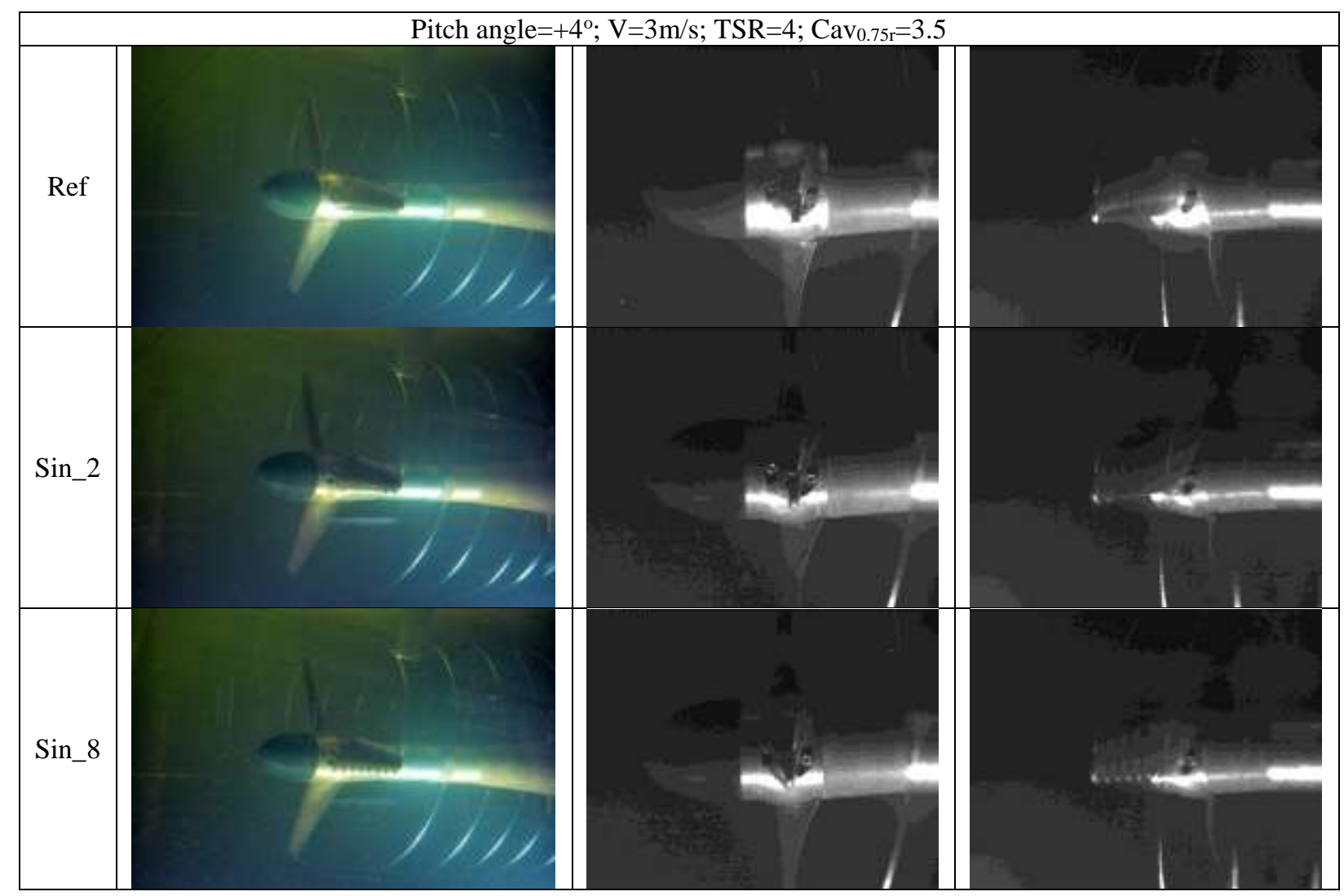

Table 8 Back side cloud cavitation

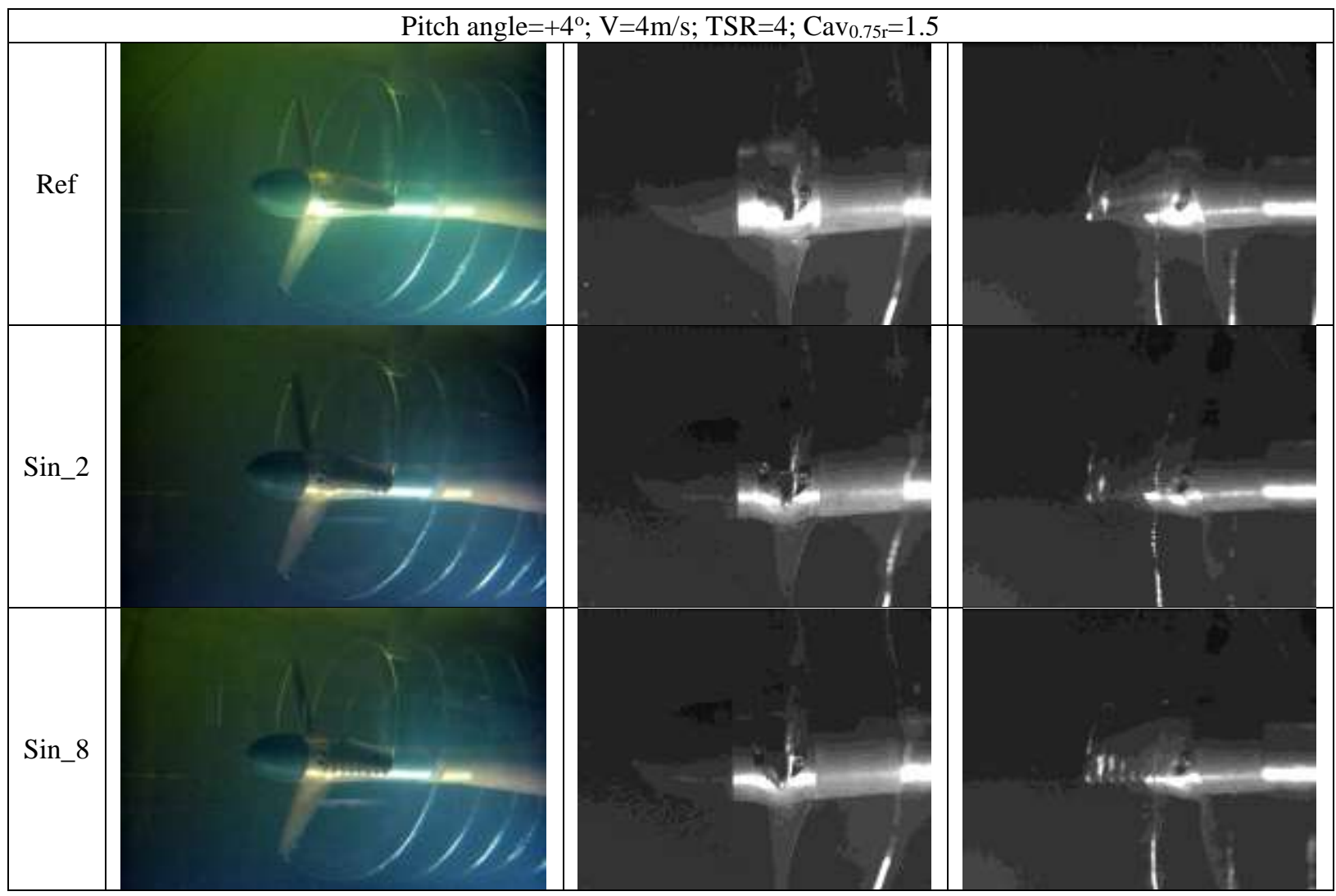


Table 9 Face side cloud cavitation

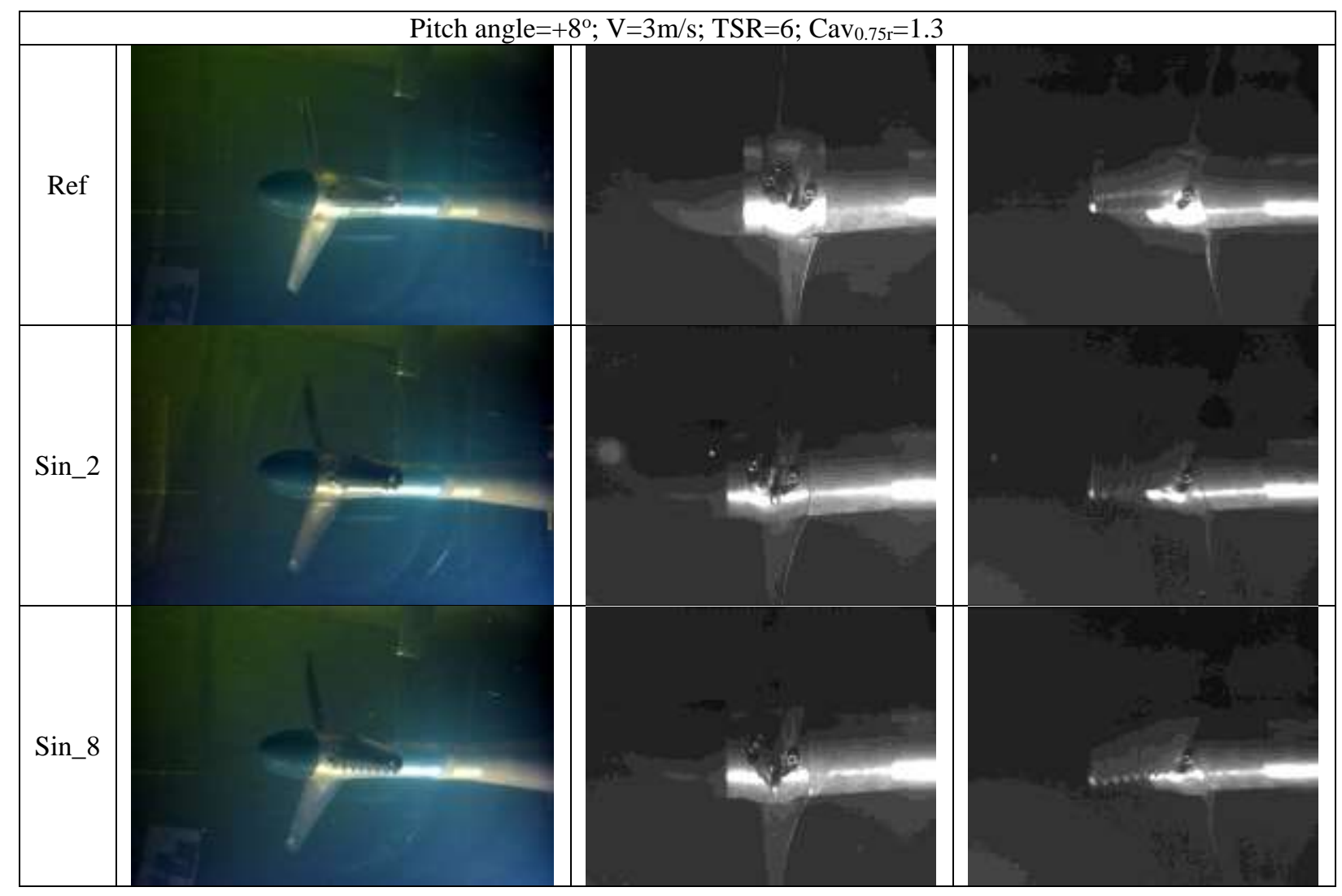

Table 10 Double side cloud cavitation

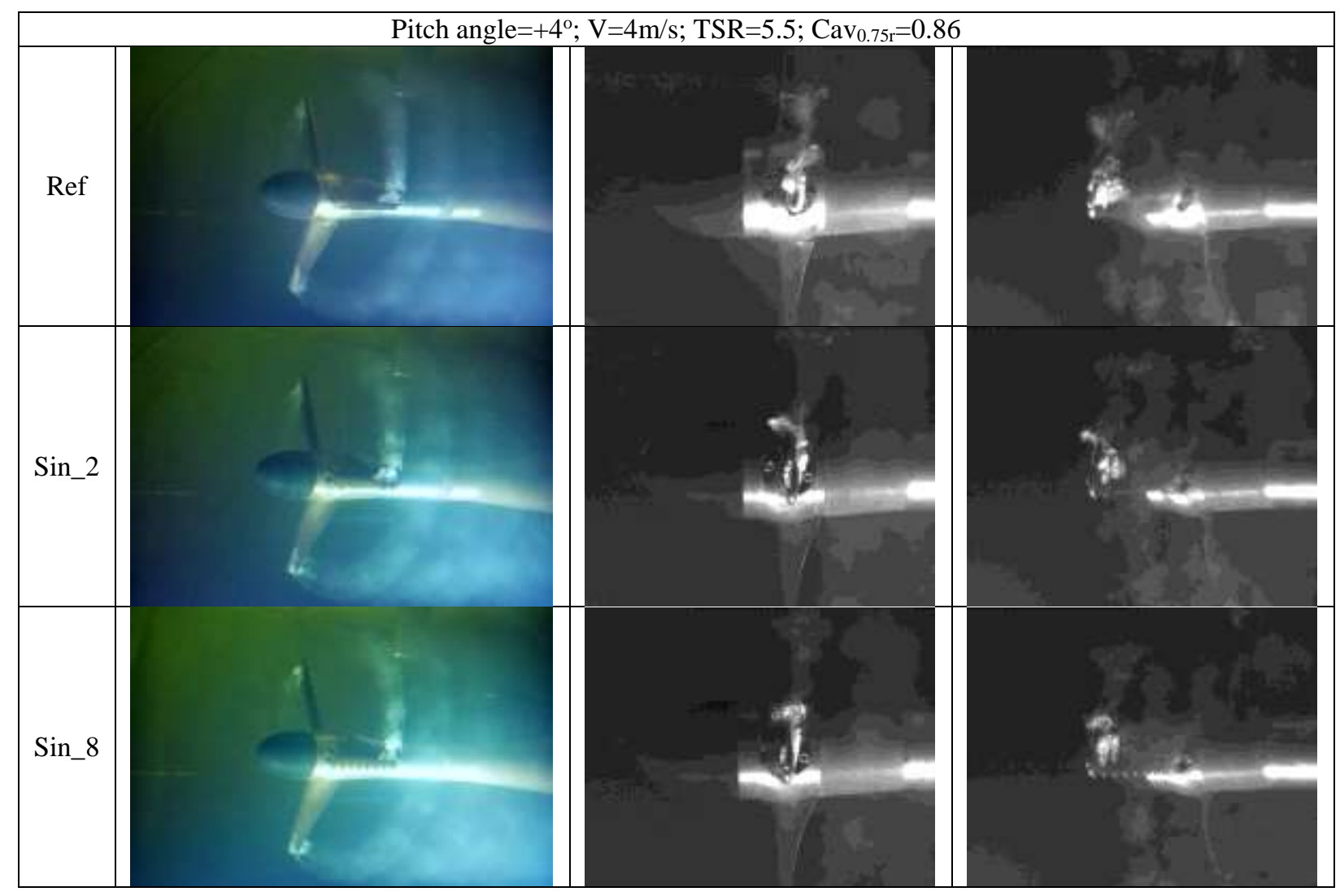

